\title{
Towards Classification of Fracton Phases: The Multipole Algebra
}

\author{
Andrey Gromov \\ Brown Theoretical Physics Center and Department of Physics, Brown University, \\ Providence, Rhode Island 02912, USA \\ and UC Berkeley and Lawrence Berkeley National Laboratory, Berkeley, California 94720, USA
}

(Received 30 January 2019; revised manuscript received 19 June 2019; published 27 August 2019)

\begin{abstract}
We present an effective field theory approach to the fracton phases. The approach is based on the notion of a multipole algebra. It is an extension of space(time) symmetries of a charge-conserving matter that includes global symmetries responsible for the conservation of various components of the multipole moments of the charge density. We explain how to construct field theories invariant under the action of the algebra. These field theories generally break rotational invariance and exhibit anisotropic scaling. We further explain how to partially gauge the multipole algebra. Such gauging makes the symmetries responsible for the conservation of multipole moments local, while keeping rotation and translations symmetries global. It is shown that upon such gauging one finds the symmetric tensor gauge theories, as well as the generalized gauge theories discussed recently in the literature. We refer to all such theories as multipole gauge theories. The outcome of the gauging procedure depends on the choice of the multipole algebra. In particular, we show how to construct an effective theory for the U(1) version of the Haah code based on the principles of symmetry and provide a two-dimensional example with operators supported on a Sierpinski triangle. We show that upon condensation of charged excitations, fracton phases of both types as well as various Symmetry-protected topological phases emerge. Finally, the relation between the present approach and the formalism based on polynomials over finite fields is discussed.
\end{abstract}

DOI: 10.1103/PhysRevX.9.031035

Subject Areas: Condensed Matter Physics

\section{INTRODUCTION}

Fracton order is a class of gapped phases of matter that exhibits a system-size-dependent ground-state degeneracy on a space of nontrivial topology. This degeneracy cannot be lifted by local perturbations that do not distort the geometry of the lattice. Another striking feature of the fracton order is the existence of local topologically nontrivial [1] excitations with "restricted mobility" [2-7]. The latter refers to the absence of stringlike operators that would allow the local excitations to move through the space without creating additional excitations. This is in sharp contrast with, for example, fractional quantum Hall states, where quasiholes can be freely transported (provided they were localized by an external potential) [8]. These models were originally introduced as an example of glassy behavior without disorder by Chamon [2] and as a model for stable quantum memory by Haah [3]. The term "fracton" has been previously used to refer to small-scale thermal

Published by the American Physical Society under the terms of the Creative Commons Attribution 4.0 International license. Further distribution of this work must maintain attribution to the author(s) and the published article's title, journal citation, and DOI. vibrations of fractal structures [10]. We hope that the present use of the term will not cause confusion.

According to the recent nomenclature [7], the fracton phases come in two varieties: type I and type II. Type-I phases support completely immobile excitations-fractons-at corners of codimension 1 surface operators (such as membranes in $d=3$ ) [2,5-7]. Various combinations of fractons can freely move around on lower-dimensional submanifolds [6,7]. Type-II phases support only immobile fracton excitations, that exist at "corners" of fractal operators, i.e., the nonlocal operators, supported on a fractal.

Despite significant research effort, it is presently not known what is the appropriate mathematical structure that encodes the exotic properties of the fracton order in a model-independent fashion. The first substantial progress in a model-independent description of fractons was made in a series of papers [11-13], where it was explained that restricted mobility of type-I models can be incorporated into an effective field theory by enforcing a certain set of Gauss law constraints. These constraints lead to the conservation of the dipole moment (or generally various multipole moments) of the matter fields. It was also noted that lattice gauge theories with such Gauss law constraints have been previously studied in Refs. [14,15]. Degrees of freedom (d.o.f.) in these theories are described by a symmetric tensor gauge field, and the Gauss law is enforced 
upon a symmetric tensor electric field. This type of effective theory does not describe a gapped phase "as is," since there are gapless excitations. A version of the Higgs mechanism was developed to remove the gapless modes in Refs. [16,17].

In a somewhat surprising parallel development it was argued that certain type-I fracton models are related to a quantum theory of elasticity [18-21]. The relation can be argued either from a duality point of view [18], where the gauge "symmetry" emerges from solving the momentum conservation equation, or starting with the observation that the gauge transformations in symmetric tensor gauge theories are identical to linearized diffeomorphisms, which is a symmetry in theories of elastic defects. Under this correspondence the immobile fractons map to disclinations, while the partially mobile fracton dipoles map onto dislocations (which satisfy the glide constraint). We note in passing that the duality in the context of elasticity has been previously studied in a series of papers by Kleinert [22,23], where (Euclidean) symmetric tensor gauge theories (of vector charge type) were introduced; however, the glide constraint was omitted. It was further noted in Ref. [19] (and later extended in Ref. [24]) that symmetric tensor gauge theories cannot remain gauge invariant in a general curved background, which is in striking difference compared to traditional electrodynamics. This observation is in correspondence with a series of works [25-28], where the effective theory of the X-cube model (a particular representative of type-I fracton models) was derived from the microscopics, studied in the presence of disclinations, and generalized to be defined on arbitrary foliated manifolds. The physical properties of these models (such as groundstate degeneracy and restricted mobility) depend on the geometry of the underlying space; thus we find it to be more appropriate to refer to fracton systems as geometric order, as suggested in Ref. [26].

In yet another parallel development it was explained that the fracton phases can be further obtained by "gauging" a subsystem symmetry [29-32]. This leads to a swarm of microscopic models. A close relative of such symmetries, known as sliding symmetry, appears in a theory of smectics (as well as other layered phases) that are often studied in soft condensed matter physics as well as in certain layered models $[33,34]$. On a lattice it is possible to define a subsystem symmetry that acts on a fractal set of lattice sites. Gauging such symmetry leads to the type-II fracton phases $[29,30]$. The long-distance description of the subsystem symmetries of this kind is not understood. Parity breaking phases of fractons, with possible gapless boundary modes, were studied in Refs. $[19,35,36]$. Further work on fracton phases and related topics can be found in Refs. [37-48], while a broad picture is given in the review [49]. Finally, we note that a similar formalism has been used to describe the geometric properties of fractional quantum Hall states. Namely, the foliated spacetime has been used in Refs. [50-53] to describe the transport of energy and momentum, while the symmetric tensor d.o.f. have appeared in the context of nematic quantum Hall states and collective magnetoroton modes [54-60].

\section{A. Summary of results}

The main objective of the present paper is to introduce a language that allows us to systematically construct a rich variety of effective field theories, which exhibit the phenomenology of both type-I and type-II fracton models. This will be done in such a way that both types appear as particular cases of the general framework.

Our construction rests upon an extension of a space(time) symmetry algebra, which we dub the multipole algebra. This algebra is a natural generalization of the symmetry algebras generated by the polynomial shift symmetries studied in Refs. [61-64]. These symmetries were originally introduced in the context of Galileon gravity [61]. In systems with a conserved U(1) charge these global symmetries lead to the conservation of the (various components of) multipole moments of the charge density. The aforementioned symmetries cannot be regarded as "internal" because they do not commute with spatial translations and rotations. These algebras are, in general, quite delicate objects, as certain consistency conditions must be satisfied. These conditions arise from the intricate interplay between the spatial and multipole symmetries. If the multipole generators are picked "at random," the algebra will only close if all spatial symmetries are discarded. In such a case all generators simply commute. A particular case of the (exponentiated) polynomial shift symmetry was also considered by Pretko in Ref. [65], where the scalar and vector gauge theories were obtained from gauging a global symmetry.

After defining the multipole algebra we explain how to construct effective field theories, invariant under its action. We restrict our attention to the matter described either by a real scalar "phase" field or by a charged complex scalar. These theories are introduced by first constructing all possible invariant derivative operators, consistent with the multipole algebra, and then including all terms allowed by the symmetries, in the effective action. Such theories usually break spatial rotations, have unusual scaling properties, and are generally quite exotic. Further, we discover that some of these theories in $d=3$ exhibit an enhanced sliding symmetry, alluded to in the Introduction.

Gauging of the multipole algebra should lead to exotic theories of elasticity and/or gravity, because in order to consistently gauge these theories one must gauge the spatial rotations and translations. This explains why symmetric tensor gauge theories (which are a particular case of the present construction) are very sensitive to the background geometry. It is, however, possible to "partially" gauge these symmetry algebras, under the assumption that the space (time) part of the curvature tensor is trivial (simply put, in flat space, without torsion). This partial gauging procedure 
leads to a very rich set of gauge theories, which includes all known symmetric tensor gauge theories (and variations thereof: higher rank; scalar, vector, or tensor charge; traceful or traceless; etc.) as well as the "generalized gauge theories" introduced in Ref. [66]. We dub such theories the multipole gauge theories. Multipole gauge theories naturally satisfy the exotic Gauss law constraints, which in the present formalism, are systematically derived by gauging the multipole algebra. These Gauss law constraints can be visualized, upon discretization on a lattice, as prescribing the "allowed" charge configurations. These charge configurations specify which excitations are mobile, which are subdimensional, and which are fractal. To be concrete, we construct the continuous model for the $\mathrm{U}(1)$ version of the Haah code [66] from the symmetry principles.

The gauge theories described above are gapless and do not correspond to the gapped fracton phases. To further advance our construction we show, building upon Ref. [16], that an extremely rich variety of phases emerges after the condensation of charge $k$ objects, which leads to the reduction in symmetry from $\mathrm{U}(1)$ to $\mathbb{Z}_{k}$. It is particularly interesting that, depending on the value of $k$, some of the allowed charge configurations become redundant, while in other cases a complex charge configuration turns into a hopping operator, which moves charges over several lattice spacings. Using this procedure we find a version of the Sierpinski triangle model in two dimensions and the $\mathbb{Z}_{2}$ Haah code. We then explain how to translate the obtained results into the language of polynomials over finite fields. We further explain how to define multipole moments over a finite field directly from the polynomials.

The paper is organized as follows. In Sec. II we introduce the generalized polynomial shift symmetries and use these symmetries to motivate the multipole algebra. Next, we define the multipole algebra in abstract terms and illustrate the definition on a couple of examples. In Sec. III we explain how to construct the invariant field theories and how to partially gauge the multipole algebra, at the level of the matter theory. We investigate several examples of such gauge theories in two and three dimensions. In Sec. IV we discuss various extensions of the multipole algebra, most notably the charge condensation and crystalline symmetries. We also explain the relation between the present formalism and the approach based on the polynomials over finite fields. Finally, in Sec. V we present our conclusions and discuss open directions.

\section{MULTIPOLE ALGEBRA}

\section{A. Polynomial shift symmetry}

The conservation of the dipole moment and its relevance to the fracton order was emphasized in Refs. $[12,46]$. The symmetries that have to do with the conservation of the multipole moments have been extensively studied prior to these works [61-64] and are known as polynomial shift symmetries. To simplify the presentation we first introduce these symmetries by specifying the action on the matter fields. To be concrete, consider a real scalar field $\varphi$. The action of the polynomial shift symmetry is defined according to

$$
\delta \varphi=\lambda_{\alpha} P^{\alpha}(x),
$$

where $\lambda^{\alpha}$ is a symmetry parameter and $P^{\alpha}(x)$ is a fixed polynomial; the sum over $\alpha$ is understood. The vector and tensor charge theories can be treated in a similar fashion. One has to introduce vector fields $\theta^{i}$ or tensor fields $\theta^{i j \ldots}$ and supplement those with various kinds of constant and polynomial shifts. The exploration of these structures is left for the future work.

We further assume that there is a finite number of transformations that appear in Eq. (1). If the dynamics of $\varphi$ is described by an action $S[\varphi]$, which is invariant under Eq. (1), then we have the following set of conserved charges:

$$
Q^{\alpha}=\int d^{d} x q^{\alpha}(x)
$$

where $q^{\alpha}(x)$ is the charge density that can be found via a direct application of Noether's theorem. If among $P^{\alpha}(x)$ there is a constant polynomial-corresponding to the global U(1) charge conservation-then we can write a more intuitive expression for the charges. Denoting the charge density as $\rho(x)$, we find

$$
Q^{\alpha}=\int d^{d} x P^{\alpha}(x) \rho(x),
$$

which implies the conservation of various generalized multipole moments. If we further assume that the polynomials $P^{\alpha}$ are homogeneous,

$$
P_{a}^{I_{a}}(x)=\sum_{i_{1}, i_{2}, \ldots, i_{a}} \mu_{i_{1} i_{2} \ldots i_{a}}^{I_{a}} x^{i_{1}} x^{i_{2}} \ldots x^{i_{a}}
$$

we can identify Eq. (3) with the (components of) proper multipole moments. To be specific,

$$
Q^{I_{a}}=\int d^{d} x \mu_{i_{1} i_{2} \ldots i_{a}}^{I_{a}} x^{i_{1}} x^{i_{2}} \ldots x^{i_{a}} \rho(x) .
$$

Notice that since the coordinates $x$ are dimensionalfull, then so are the parameters $\lambda_{\alpha}$. This ultimately leads to the generalized Mermin-Wagner theorem, which states that if the power of the polynomials in Eq. (1) is no larger than $n$, then the symmetry cannot be spontaneously broken in $d \leq$ $n+1$ spatial dimensions [64]. This is quite similar to the Mermin-Wagner theorem for the higher form symmetry [67]. The relation between these two types of symmetry warrants further exploration. 
A particularly simple case of this structure is the symmetry under all polynomial shifts of the degree no greater than $n$. Such transformation takes the form

$$
\delta \varphi=\lambda+\lambda_{i}^{(1)} x^{i}+\lambda_{i j}^{(2)} x^{j} x^{j}+\cdots .
$$

This leads to the conservation of all multipole moments of degree less or equal to $n$. The conserved charges are the arbitrary moments of the density $Q^{i j \ldots}=\int d^{d} x \rho(x) x^{i} x^{j} \ldots$. The case of $n=1$ corresponds to the conservation of the dipole moment and leads to the scalar charge theory [12], while the restricted mobility of dipoles can be added by supplementing the $n=1$ symmetry with $\delta \varphi=\lambda^{\prime}|x|^{2}$, which leads to the traceless scalar charge theory.

\section{B. Multipole algebra}

The transformations Eq. (1) commute with each other and form an unremarkable algebraic structure. However, these transformations do not commute with the spatial symmetries: translations and rotations. Instead, the polynomial shift symmetries extend the algebra of spatial symmetries to a bigger multipole algebra $\mathfrak{m}$. Consequently, the symmetries responsible for the conservation of the multipole moments are not internal. This provides a general explanation to the observation made in Ref. [19] (and later extended in Ref. [24]) that the symmetric tensor gauge symmetry is "broken" on a general curved manifold.

\section{Intuitive preamble}

Before diving into the formal details of the multipole algebra, we would like to illustrate the physical origins of the structure. Consider a transformation law of a quadrupole moment of the charge density under a translation by the vector $r^{k}$,

$$
\delta q^{i j}=r^{j} q^{i}+r^{i} q^{j}+r^{i} r^{j} q,
$$

where $q^{i}$ is the total dipole moment and $q$ is the total charge. Usually, one would state that provided that all lower moments vanish, the quadrupole moment is invariant under translations. Imagine a situation when $q^{1}=q^{2}=0$ is enforced by the symmetry and all other components of the dipole moment are nonzero. Then the quadrupole moment is invariant under translations in the $x_{1}-x_{2}$ plane, provided that the total charge $q$ vanishes. Thus we can further constrain the quadrupole moment by symmetries defined within this plane.

We will further encounter another interesting degenerate case, which plays the central role in the discussion of the fractal phases. Consider a particular component of the quadrupole moment $q_{\mu}=q^{i j} \mu_{i j}$, where $\mu_{i j}$ is a certain degenerate matrix with the property that its kernel equals to the orthogonal complement of the $x_{1}-x_{2}$ plane. Then such a component of the quadrupole moment is translationally invariant under all translations:

$$
\delta q_{\mu}=\mu_{i j} r^{j} q^{i}+\mu_{i j} r^{i} q^{j}+\mu_{i j} r^{i} r^{j} q=0 .
$$

This equality holds for all translations since if we take translations outside of the $x_{1}-x_{2}$ plane, the matrices $\mu_{i j}$ will project them down to the $x_{1}-x_{2}$ plane, but in this plane the projection of the total dipole moment vanishes. Thus $q_{\mu}$ is translation invariant.

The multipole algebra formalizes these simple ideas in the language of symmetry.

\section{Formal definition of the multipole algebra}

To get some insight into the algebraic structure, we need to sort the polynomials by their degree. We introduce a set of generators of the polynomial symmetries $\mathcal{P}_{a}^{I_{a}}$, so that $a$ is the degree of the polynomial, whereas $I_{a}$ runs through all polynomials of degree $a$. Then the general multipole algebra is defined via the following set of commutation relations:

$$
\begin{aligned}
& {\left[R_{i j}, T_{k}\right]=\delta_{k[i} T_{j]}, \quad\left[R_{i j}, R_{k l}\right]=\delta_{[k[i} R_{j] l]},} \\
& {\left[R_{i j}, \mathcal{P}_{a}^{I_{a}}\right]=f_{i j}{ }_{a}{ }_{a}{ }_{a}{ }_{J_{b}} \mathcal{P}_{b}^{J_{b}}, \quad\left[T_{i}, \mathcal{P}_{a}^{I_{a}}\right]=f_{i}^{I_{a}{ }_{a}{ }_{J_{b}}} \mathcal{P}_{b}^{J_{b}},}
\end{aligned}
$$

where $T_{i}$ and $R_{i j}$ are the generators of spatial translations and rotations, $O_{[i j]}$ denotes antisymmetrization over $i, j$, and $f_{i j}{ }_{i j}{ }_{a}{ }_{a} J_{b}, f_{i} I_{a}{ }_{a}{ }^{b} J_{b}$ are the structure constants that define the algebra. In the polynomial representation the content of Eq. (10) is quite simple: rotations and translations, when applied to the polynomials, should produce linear combinations of the polynomials within the multipole algebra; i.e., the set of polynomials $P_{a}^{I_{a}}$ is closed under rotations and translations. The multipole algebra can be exponentiated to a multipole group $\mathfrak{M}$. The multipole group is, in a way, a more fundamental construct since, unlike the algebra, it admits a crystalline analogue and survives the condensation of charge $p$ objects (provided the charge is well defined) as a discrete group.

If the polynomials depend on $d$ variables, then the indices $i, j$ run from 1 to $k \leq d$. This happens because generally the vector space of polynomials spanned by $\mathcal{P}_{a}^{I_{a}}$ is not compatible with translations or rotations; in such case the multipole algebra is trivial. However, if $\mathcal{P}_{a}^{I_{a}}$ are chosen carefully, then very rich and intricate structures can emerge.

\section{Maximal multipole algebra}

Next we turn to consider a few explicit examples. The simplest case is the set of transformations Eq. (6) with $n=1$. It gives rise to the following algebra [we omit the usual relations between translations and rotations (9)]:

$$
\left[T_{i}, \mathcal{P}_{1}^{j}\right]=\delta_{i j} \mathcal{P}^{0}, \quad\left[R_{i j}, \mathcal{P}_{1}^{k}\right]=\delta_{k[i} \mathcal{P}_{1}^{j]},
$$


where $\mathcal{P}^{0}$ refers to the constant shifts. We emphasize that the internal index $I$ was identified with the spatial index $i$. This is due to an "accidental" isomorphism between the set of linear shifts and the set of translations. One distinguishing feature of this algebra is that is it consistent with all spatial translations and rotations. This happens because all polynomials of degree $\leq 1$ were included; consequently, such a set is closed under any operation that does not increase the power of the polynomial.

One simple extension of the algebra Eq. (11), consistent with all spatial symmetries, is the addition of one extra generator $\mathcal{P}_{2}^{0}$, corresponding to $\delta \varphi=\lambda^{\prime}|x|^{2}$. This generator leads to the commutation relations

$$
\left[T_{i}, \mathcal{P}_{2}^{0}\right]=2 \mathcal{P}_{1}^{i}, \quad\left[R_{i j}, \mathcal{P}_{2}^{0}\right]=0 .
$$

We note in passing that the generators $\left\{T_{i}, R_{i j}, \mathcal{P}^{0}, \mathcal{P}_{1}^{i}, \mathcal{P}_{2}^{0}\right\}$ form the Bargmann algebra [68], where the spatial translations $T_{i}$ correspond to the Galilean boosts, linear shifts $\mathcal{P}_{1}^{i}$ correspond to the spatial translations, $\mathcal{P}_{2}^{0}$ to the Hamiltonian, and $\mathcal{P}^{0}$ to the mass central charge. It would be interesting to explore this isomorphism to construct field theories invariant under this type of multipole algebra.

In the above set of examples it is amusing to note that the commutation relations between $R_{i j}$ and $\mathcal{P}_{1}^{k}$ are the same as between $R_{i j}$ and $T_{k}$. In the absence of the generator $\mathcal{P}_{2}^{0}$, which provides the asymmetry, we could swap $T_{k}$ and $\mathcal{P}_{1}^{k}$ and obtain the same algebra back. This observation has a nice elastic interpretation. If the theory of elasticity is viewed as a gauge theory of translations and rotations, $T_{d} \rtimes \mathrm{SO}(d)$, then we could use either real translations or shift symmetries to construct such a gauge theory. This ambiguity corresponds to the two distinct approaches to the relationship between fractons and elasticity $[18,19]$. If the generator $\mathcal{P}_{0}^{2}$ is introduced and gauged, then one will end up with two different theories. We will elaborate on this distinction in a forthcoming work.

These symmetric cases can be generalized to arbitrary multipole moments. In order to include the multipole moments up to $a$ in $d$ spatial dimensions, we introduce a set of polynomial symmetries described by arbitrary symmetric tensors of degree $a, \mathcal{P}_{a}^{I_{a}}=\mathcal{P}_{a}^{i_{1} \ldots i_{a}}$. Each such tensor has $\left(\begin{array}{c}d+a-1 \\ a\end{array}\right)$ independent components. Together with translations and rotations these symmetry generators form the following algebra:

$$
\begin{aligned}
{\left[T_{j}, \mathcal{P}_{a}^{i_{1} i_{2} \ldots i_{a}}\right] } & =\mathcal{P}_{a-1}^{i_{1} \ldots i_{m-1} i_{m+1} \ldots i_{a}} & & \text { if } j=i_{m} \\
& =0 & & \text { otherwise, } \\
{\left[R_{j l}, \mathcal{P}_{a}^{i_{1} i_{2} \ldots i_{a}}\right] } & =\delta_{\left[j \left[i_{1}\right.\right.} \mathcal{P}_{a}^{\left.l] i_{2}\right] \ldots i_{a}} & & \text { if } j=i_{m}, l=i_{m^{\prime}} \\
& =0 & & \text { otherwise. }
\end{aligned}
$$

Theories that are symmetric under this algebra conserve all the multipole moments up to order $n$. Such multipole algebras are completely characterized by the spatial dimension and a single integer $n$; thus we refer to those as maximal multipole algebra of order $n, \mathfrak{m}_{\max }^{n}$. It may be tempting to speculate [12] that the theory obtained by gauging the algebra Eq. (13) (perhaps in the limit $n \rightarrow \infty$ ) may be related to the Vassiliev theories of higher spin gravity. In view of the algebra, Eqs. (13) and (14), this speculation seems unlikely since the symmetric multipole generators all commute with each other, whereas in the Vassiliev higher spin theory they form a version of the $W_{\infty}$ algebra [also referred to as $\operatorname{sh} s(1)$ in earlier works [69]].

\section{Homogeneous multipole algebra}

Next we turn to a less symmetric, but still very palatable case. Consider a set of polynomial symmetries where all polynomials $P_{a}^{I_{a}}$ are homogeneous, that is, of the form Eq. (4). We refer to such multipole algebras as homogeneous. We would like to demonstrate how certain rotation and translation generators drop form the algebra due to the requirement that the set of polynomials $P_{a}^{I_{a}}$ is closed under as many translations and rotations as possible. Consider, for example,

$$
\delta \varphi=\sum_{a, I_{a}} \lambda_{I_{a}} P_{a}^{I_{a}}(x),
$$

where the five polynomials $P_{a}^{I_{a}}(x)$ are given by

$$
\begin{gathered}
P_{0}^{0}=1, \quad P_{1}^{1}=\mu_{i}^{1} x^{i}, \quad P_{1}^{2}=\mu_{i}^{2} x^{i}, \\
P_{2}^{1}=\mu_{i j}^{1} x^{i} x^{j}, \quad P_{2}^{2}=\mu_{i j}^{2} x^{i} x^{j},
\end{gathered}
$$

where $\mu_{i}^{I_{1}}$ and $\mu_{i j}^{I_{2}}$ are fixed rank-1 and rank-2 tensors and $i, j=1, \ldots, d$. These tensors are fixed up to the freedom of replacing $\mu_{i}^{I_{1}}$ and $\mu_{i j}^{I_{2}}$ with the linear combinations thereof.

Depending on the circumstances, these polynomials can give rise to several multipole algebras. First, assume that $\mu_{i j}^{I_{2}}$ are all nondegenerate. Then only two out of $d$ translations survive. These are translations in the directions $t_{(1)}^{j}$ and $t_{(2)}^{j}$, which are explicitly determined from

$$
\begin{aligned}
& \mu_{i j}^{1} t_{(\alpha)}^{j}=\alpha_{1} \mu_{i}^{1}+\alpha_{2} \mu_{i}^{2}, \\
& \mu_{i j}^{2} t_{(\alpha)}^{j}=\beta_{1} \mu_{i}^{1}+\beta_{2} \mu_{i}^{2},
\end{aligned}
$$

where $\alpha_{i}, \beta_{i}$ are some constants.

Next we consider rotations. Clearly, the only rotation generator that has a chance to survive is the rotation in the $\mu_{i}^{1}-\mu_{j}^{2}$ plane, $R_{12}$. The fate of $R_{12}$ depends on the projection of $\mu_{i j}^{I_{2}}$ to the $\mu^{1}-\mu^{2}$ plane. If either the projection has nonzero trace, then the rotation invariance is lost. If, however, the projection is a linear combination of the traceless Pauli matrices $\sigma^{1}$ and $\sigma^{3}$, then, within this plane, we can always arrange that 


$$
\left[R_{12}, \mu_{i j}^{1}\right]=\mu_{i j}^{2}, \quad\left[R_{12}, \mu_{i j}^{1}\right]=-\mu_{i j}^{2},
$$

via redefining the symmetry transformations in such a way that, within the $\mu_{i}^{1}-\mu_{j}^{2}$ plane, $\mu_{i j}^{1} \propto \sigma^{1}$ and $\mu_{i j}^{2} \propto \sigma^{3}$. Thus we obtain the multipole algebra,

$$
\begin{gathered}
{\left[T_{1}, \mathcal{P}_{1}^{I_{1}}\right]=\left[T_{2}, \mathcal{P}_{1}^{I_{1}}\right]=\mathcal{P}_{0}^{0},} \\
{\left[T_{1}, \mathcal{P}_{2}^{I_{2}}\right]=f_{1 I_{1}}^{I_{2}} \mathcal{P}_{1}^{I_{1}}, \quad\left[T_{2}, \mathcal{P}_{2}^{I_{2}}\right]=f_{2 I_{1}}^{I_{2}} \mathcal{P}_{1}^{I_{1}},} \\
{\left[R_{12}, \mathcal{P}_{2}^{I_{2}}\right]=\epsilon_{J_{2}}^{I_{2}} \mathcal{P}_{2}^{J_{2}},}
\end{gathered}
$$

where sum over repeated $I_{a}, J_{a}$ is understood. Although we have started in $d$ spatial dimensions, only two translation generators and one rotation generator have survived.

There is another interesting possibility, which will arise in the study of the U(1) Haah code: both $\mu_{i j}^{I_{2}}$ are degenerate in such a way that the kernels of $\mu_{i j}^{I_{2}}$ coincide with each other and with the orthogonal complement of $\mu_{i}^{I_{1}}$. Then Eqs. (18) and (19) hold true for all translations since $\mu_{i j}^{I_{2}}$ are projectors to the $\mu_{i}^{1}-\mu_{i}^{2}$ plane. In such algebra we get an additional set of trivial commutation relations:

$$
\left[T_{i}, \mathcal{P}_{2}^{I_{2}}\right]=0
$$

where $i$ runs over the orthogonal complement to $\mu_{i}^{I_{1}}$ : from 1 to $d-2$ in the present example.

\section{INVARIANT FIELD THEORIES}

\section{A. General constraints}

We turn to the construction of the field theories invariant under the action of the multipole algebra. First, we fix a multipole algebra $\mathfrak{m}$ and construct an irreducible representation. To start, we have to fix the transformation law under rotations. For simplicity we take a single real scalar field $\varphi$ [70]. The transformation laws under the action of $\mathcal{P}_{a}^{I_{a}}$ are given by Eq. (1). We denote the highest power that appears in Eq. (1) as $a_{\max }$. The time derivative term takes the ordinary form, $\dot{\varphi} \dot{\varphi}$ [71]. To construct the kinetic term, we need an invariant derivative operator, i.e., a derivative operator, consistent with Eq. (1). To this end we consider a general differential operator

$D=q+q^{i_{1}} \partial_{i_{1}}+q^{i_{1} i_{2}} \partial_{i_{1}} \partial_{i_{2}}+\cdots+q^{i_{1} \ldots i_{s}} \partial_{i_{1}} \ldots \partial_{i_{s}}$,

where $s \leq a_{\max }$. The coefficients $q^{i_{1} i_{2} \ldots}$ will be chosen in such a way that $D \varphi$ is invariant under the action of $\mathcal{P}_{a}^{I_{a}}$. In the most general case, these equations take the form

$$
D P_{a}^{I_{a}}=0,
$$

where $P_{a}^{I_{a}}$ is the polynomial corresponding to the action of $\mathcal{P}_{a}^{I_{a}}$. Equation (26) must hold for all $P_{a}^{I_{a}}$. The solutions to these equations are the differential operators $D_{\alpha}$ (the index $\alpha$ labels the solutions). The constraints Eq. (26) can be written more explicitly if we introduce the following parametrization for $P_{a}^{I_{a}}(x)$ :

$$
P_{a}^{I_{a}}(x)=\mu^{I_{a}}+\mu_{i_{1}}^{I_{a}} x^{i_{1}}+\cdots+\mu_{i_{1} \ldots i_{a}}^{I_{a}} x^{i_{1}} \ldots x^{i_{a}} .
$$

Then Eq. (26) turns into a set of linear equations on $q^{i_{1} \ldots}$,

$$
\begin{gathered}
q \mu^{I_{0}}+q^{i} \mu_{i}^{I_{1}}+q^{i j} \mu_{i j}^{I_{2}}+\cdots=0, \\
q \mu_{i_{1}}^{I_{1}}+q^{i} \mu_{i_{1} i}^{I_{2}}+q^{i j} \mu_{i_{1} i j}^{I_{3}}+\cdots=0, \\
\ldots, \\
q \mu_{i_{1} \ldots i_{a-1}}^{I_{a-1}}+q^{i} \mu_{i_{1} \ldots i_{a}}^{I_{a}}=0, \\
q \mu_{i_{1} \ldots i_{a}}^{I_{a}}=0,
\end{gathered}
$$

which hold for all $I_{a}$. It immediately follows that $q=0$ (otherwise all $\mu^{I_{a}}$ have to vanish). Thus, the invariant derivatives must start with at least $q^{i_{1}} \partial_{i_{1}}$. This implies an additional invariance of the effective theory under a global $\mathrm{U}(1)$ transformation, $\delta \varphi=\mu^{I_{0}}$.

The system Eqs. (28)-(31) contains $\{[(2 a) !] / a !\}$ equations for every polynomial, and only $\{[(2 a) !] / a !\}$ unknowns. Thus, generally it is severely overdetermined and has no solutions. As we will see shortly, there are, indeed, "degenerate" cases when the system does admit solutions. This phenomenon is somewhat reminiscent of the existence of a solution to the (severely overdetermined) pentagon and hexagon equations, which are the consistency conditions for fusion tensor categories.

If there are no solutions for $s \leq a_{\max }$, then the system can always be solved by a higher-order differential operator that annihilates all polynomials of degree no greater than $a_{\max }$. Such an operator takes the form

$$
D=q^{i_{1} \ldots i_{a_{\max }+1}} \partial_{i_{1}} \ldots \partial_{i_{a_{\max }+1}},
$$

for any $q^{i_{1} \ldots i_{a_{\max }+1}}$. The action constructed using these solutions will have an enhanced symmetry under all polynomial shifts and will represent the maximal multipole algebra $\mathfrak{m}_{\max }^{a_{\max }}$. Presently it is not clear how to establish the existence of a solution to Eqs. (28)-(31) without solving $O\left(4^{a} a^{a}\right)$ equations.

A comment is in order. In the above construction, as well as in the remainder of the paper, we demand the complete invariance of the action under the symmetries. It is, however, possible to consider a weaker condition - the invariance of the action up to a total derivative. This condition allows for a wider array of the invariant Lagrangians. We leave exploration of this scenario for future work. 


\section{B. Constraints in the homogeneous case}

In the remainder of the paper we focus on the homogeneous multipole algebras. The constraint equations take the form

$$
\begin{gathered}
\mu_{i}^{I_{1}} q^{i}=0, \\
\mu_{i j}^{I_{2}} q^{i}=0, \quad \mu_{i j}^{I_{2}} q^{i j}=0, \\
\mu_{i j k}^{I_{3}} q^{i}=0, \quad \mu_{i j k}^{I_{3}} q^{i j}=0, \quad \mu_{i j k}^{I_{3}} q^{i j k}=0, \\
\ldots . .
\end{gathered}
$$

The system of equations (33)-(35) is more overdetermined than Eqs. (28)-(31), since the contractions such as $\mu_{i j k}^{I} q^{i j}=0$ must vanish separately. Nevertheless, as we will see shortly, these systems still admit solutions.

Finally, we note that the solutions of Eqs. (33)-(35) are determined up to an overall scale [which is not the case for Eqs. (28)-(31)].

To the lowest order in derivatives, the invariant effective action is

$$
S=\int d^{d} x d t\left[\dot{\varphi} \dot{\varphi}-\sum_{\alpha, \beta} \lambda_{\alpha \beta}\left(D_{\alpha} \varphi\right)\left(D_{\beta} \varphi\right)\right],
$$

where $\alpha$ runs over all solutions of Eq. (26).

To the lowest order in gradients, the effective action is quadratic in the invariant derivatives. However, the latter are not necessarily of the same degree.

In the case of the maximal multipole algebra $\mathfrak{m}_{\max }^{n}$ it is possible to write a rotationally invariant action,

$S=\int d^{d} x d t\left[\dot{\varphi} \dot{\varphi}-\sum_{k>1} \lambda_{k}\left(D_{i_{1} i_{2} \ldots i_{n}} \varphi D^{i_{1} i_{2} \ldots i_{n}} \varphi\right)^{k}\right]$,

where $D_{i_{1} i_{2} \ldots i_{n}}=\partial_{i_{1}} \ldots \partial_{i_{n}}$. Rotationally invariant actions of the type Eq. (37) were studied in Refs. [61-64]. We view the actions Eqs. (36) and (37) within the framework of the effective field theory. In particular, this entails including all possible terms, consistent with the postulated symmetries, and organizing these terms according to some degree of "relevance."

\section{Multipole gauge theory}

In this section we explain how to gauge the multipole symmetries. With the invariant derivatives at hand it is now possible to introduce a local version of the multipole symmetry. In principle, we could do it directly from the commutation relations; however, this entails gauging the spatial symmetries as well. We leave this program to a future work.

The present approach allows us to "partially gauge" the symmetries: the multipole symmetries will become local, while rotations and translations will remain global. This amounts to setting the corresponding gauge fields to 0 , so that Ricci curvature and torsion vanish. The gauging procedure is well known-we promote the global symmetry in Eq. (1) to a local one $\delta \varphi=\zeta(x)$ and introduce a covariant derivative operator,

$$
\nabla_{\alpha} \varphi=D_{\alpha} \varphi+a_{\alpha}(t, x), \quad \delta a_{\alpha}(t, x)=-D_{\alpha} \zeta,
$$

where $a_{\alpha}(t, x)$ is the gauge field. The time derivative is replaced with the ordinary $\nabla_{0} \varphi=\dot{\varphi}+\chi$, with $\delta \chi=-\partial_{0} \zeta$.

With the gauge fields at hand we introduce a set of conjugate momenta (or "electric fields") according to

$$
\left[e_{\beta}\left(x^{\prime}\right), a_{\alpha}(x)\right]=-i \delta\left(x-x^{\prime}\right) \delta_{\alpha \beta} .
$$

In the present approach we construct the "magnetic fields" on a case-by-case basis as gauge-invariant combinations of $a_{\alpha}$.

The general invariant Lagrangian takes the form

$$
\mathcal{L}=\mathcal{L}\left[\nabla_{\alpha} \varphi\right]+\sum_{\alpha} e_{\alpha} \dot{a}_{\alpha}-\mathcal{H}[e, b]
$$

where $\mathcal{H}[e, b]$ schematically denotes the Hamiltonian for the gauge fields. Integrating out the nondynamical variable $\chi$ leads to the Gauss law constraint,

$$
D_{\alpha}^{\dagger} e_{\alpha}=\rho,
$$

where the conjugate derivative $D_{\alpha}^{\dagger}$ is defined as

$$
\int d^{d} x d t f\left(D_{\alpha} g\right)=\int d^{d} x d t\left(D_{\alpha}^{\dagger} f\right) g .
$$

The Gauss law of the type Eq. (41) was postulated in Ref. [66]. We find that Eq. (41) follows directly from the underlying structure of the multipole algebra.

The invariant derivatives $D_{\alpha}$ can be discretized on a lattice, leading to various charge configurations. To be concrete (see Ref. [66]), consider an operator $e^{-i a_{\alpha}(x)}$, acting on a site with label $x$. This operator changes the value of the electric field $e_{\alpha}(x)$ at the same lattice site, say, raises it by 1 . This, in turn, requires us to introduce electric charges at all lattice sites $x^{\prime}$, that are connected to $e_{\alpha}(x)$ by the Gauss law Eq. (41). We make heavy use of this pictorial representation.

Such configurations of finite number of point charges are characterized by a set of multipole moments. These moments are determined by $q_{\alpha}^{i}, q_{\alpha}^{i j}, \ldots$. Only the lowest of these moments is independent of the choice of coordinate origin; however, the solution of Eqs. (33)-(35) is independent of the choice of origin. Indeed, assume that the total charge is zero, then the dipole moment is well defined. When the origin is shifted by $r^{i}$ the quadrupole moment 
transforms as $\delta q_{\alpha}^{i j}=r^{i} q_{\alpha}^{j}+r^{j} q_{\alpha}^{i}$, which still satisfies Eqs. (33)-(35). In the most general, nondegenerate case the solutions are invariant only under the translations in "allowed" directions, such as the ones specified by Eqs. (18) and (19). This is not too surprising since other translations do not belong to the symmetry algebra.

\section{Maximally symmetric gauge theory}

Next we consider a few examples of the general formalism outlined above. First, we would like to make sure that the symmetric tensor gauge theories follow. This is indeed so, provided we gauge the maximal algebra $\mathfrak{m}_{\max }^{n}$. The covariant derivative takes the form

$$
\nabla_{i_{1} i_{2} \ldots i_{n+1}} \varphi=\partial_{i_{1}} \partial_{i_{2}} \ldots \partial_{i_{n+1}} \varphi+a_{i_{1} i_{2} \ldots i_{n+1}} .
$$

In this case a rotationally invariant action is possible:

$$
S=\int d^{d} x d t\left[\nabla_{0} \varphi \nabla_{0} \varphi-\nabla_{i_{1} i_{2} \ldots i_{n+1}} \varphi \nabla^{i_{1} i_{2} \ldots i_{n+1}} \varphi\right] .
$$

This type of (ungauged) action has been studied in great detail in Refs. [62-64], in relation to "slow" Goldstone bosons.

Including, additionally, pure trace generators of one higher degree $\mathcal{P}_{n+1}^{I_{n+1}}$ necessitates the change in the covariant derivative,

$$
\nabla_{\alpha} \varphi=q_{\alpha}^{i_{1} i_{2} \ldots i_{n+1}} \partial_{i_{1}} \partial_{i_{2}} \ldots \partial_{i_{n+1}} \varphi+a_{\alpha},
$$

where $q_{\alpha}^{i_{1} i_{2} \ldots i_{n}}$ are symmetric traceless tensors and the gauge field $a_{\alpha}$ can be related to the symmetric tensor gauge field via projecting out the traceful parts $a_{\alpha}=q_{\alpha}^{i_{1} i_{2} \ldots i_{n+1}} a_{i_{1} i_{2} \ldots i_{n+1}}$. Both traceless and traceful scalar charge theories exhibit a scaling symmetry with dynamical critical exponent $z=$ $n+1$ :

$$
t \rightarrow \lambda^{z} t, \quad x_{i} \rightarrow \lambda x_{i}, \quad \varphi \rightarrow \lambda^{(n+1-d) / 2} \varphi .
$$

Thus, in $d$ spatial dimensions, the field $\varphi$ is dimensionless if $n+1=d$. As discussed in Ref. [64], this corresponds to a generalized version of the Mermin-Wagner theorem: the (maximal) multipole symmetry of degree $n$ cannot be spontaneously broken in $d \leq n+1$ dimensions.

In the case $n=1$ we get the effective theory for the traceless scalar charge theories,

$S=\int d^{d} x d t\left[\nabla_{0} \varphi \nabla_{0} \varphi-\left(q^{i_{1} i_{2}} \nabla_{i_{1} i_{2}} \varphi\right)\left(q^{j_{1} j_{2}} \nabla_{j_{1} j_{2}} \varphi\right)+\cdots\right]$,

where $q^{i j}$ are symmetric traceless tensors. The charge configurations for these theories have been discussed previously [12]. We use this case as the first benchmark (a)

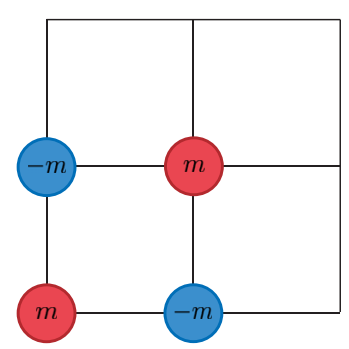

(b)

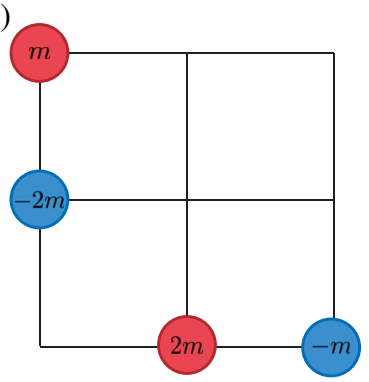

(c)
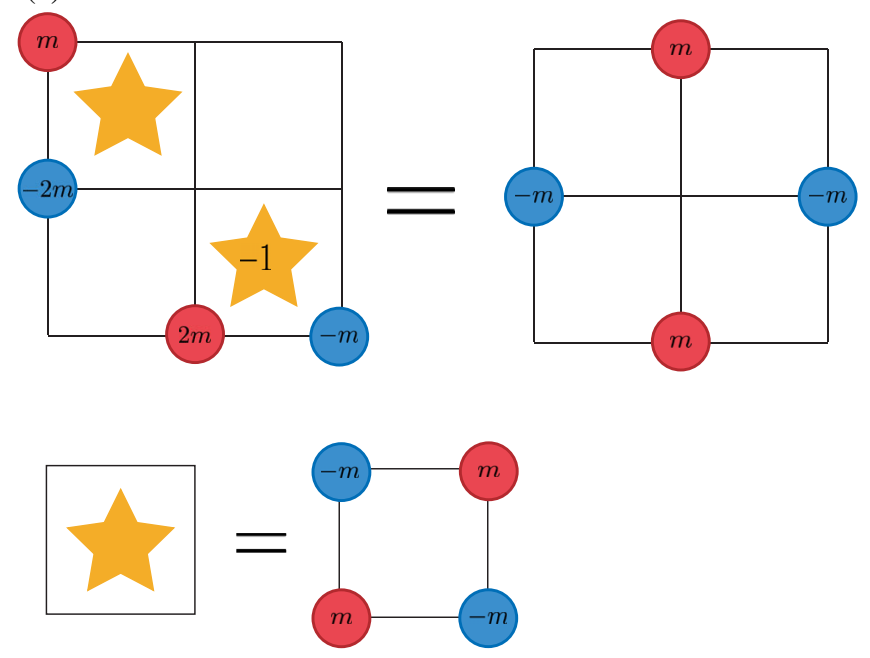

FIG. 1. (a) The charge configuration corresponding to $q^{i j} \propto \sigma^{1}$. (b) The charge configuration corresponding to $q^{i j} \propto \sigma^{3}$. (c) $\mathrm{A}$ more convenient basis of charge configurations is obtained by applying (a) and inverse of (a) at plaquettes labeled by star and -1 star correspondingly.

of the present formalism. In two dimensions the Gauss law takes the form

$$
D_{1}^{\dagger} e_{1}+D_{2}^{\dagger} e_{2}=\rho,
$$

since there are only two symmetric traceless tensors in $d=2$, namely, the Pauli matrices $\sigma_{i j}^{1}$ and $\sigma_{i j}^{3}$. The corresponding charge configurations are illustrated in Fig. 1.

\section{E. Quadratic multipole algebras in two dimensions}

In two spatial dimensions, while restricting ourselves to quadratic order, we can solve the problem of classification of homogeneous multipole algebras. The constraint equations are

$$
q^{i} \mu_{i}^{I_{1}}=0, \quad q^{i} \mu_{i j}^{I_{2}}=0, \quad q^{i j} \mu_{i j}^{I_{2}}=0 .
$$

We consider the solutions on a case-by-case basis. The first case is when $\mu_{i j}^{I_{2}}$ are nondegenerate. Then $q^{i}=0$ and the $\mu_{i}^{I_{1}}$ are arbitrary, which implies that the dipole moment is conserved. We have to consider a few possibilities for $q^{i j}$. 
To start, we parametrize the symmetric tensors using Pauli matrices and the identity matrix,

$$
\mu^{I_{2}}=\gamma_{\nu}^{I_{2}} \tau^{\nu}, \quad q=\beta_{\nu} \tau^{\nu},
$$

where $\tau_{\nu}=\left(\sigma_{0}, \sigma_{1}, \sigma_{3}\right), \nu=1,2,3$, and $\sigma_{0}$ is the identity matrix. The corresponding polynomials take the form

$$
P_{2}^{I_{2}}=\gamma_{1}^{I_{2}}\left(x_{1}^{2}+x_{2}^{2}\right)+2 \gamma_{2}^{I_{2}} x_{1} x_{2}+\gamma_{3}^{I_{2}}\left(x_{1}^{2}-x_{2}^{2}\right) .
$$

The nontrivial constraints from Eq. (49) then take the form

$$
\gamma_{\nu}^{I_{2}} \beta^{\nu}=0
$$

where $I_{2}=1, \ldots, k$. The number of solutions of Eq. (52) is $3-k$. If there are no quadratic symmetries, we find the usual symmetric tensor gauge theory, associated to the maximally symmetric multipole algebra of order 1 . If $k=1$ and $\gamma_{\nu}^{1}=\delta_{1, \nu}$, we find the symmetric traceless gauge theories. These two examples are rotationally invariant with the multipole algebra of the form

$$
\begin{aligned}
& {\left[T_{i}, \mathcal{P}_{1}^{I_{1}}\right]=\mathcal{P}_{0}, \quad\left[T_{i}, \mathcal{P}_{2}^{0}\right]=\delta_{i I_{1}} \mathcal{P}_{1}^{I_{1}},} \\
& {\left[R, \mathcal{P}_{1}^{I_{1}}\right]=\epsilon_{J_{1}}^{I_{1}} \mathcal{P}_{1}^{J_{1}}, \quad\left[R, \mathcal{P}_{2}^{0}\right]=0,}
\end{aligned}
$$

where $R$ is the only rotation generator and $T_{i}$ are the translation generators. As mentioned previously, in these cases it is convenient to use $\delta_{i I_{1}}$ to identify the spatial indices with the multipole index $I_{1}$. With this identification, the corresponding gauge fields are proper tensors.

In all other cases we find theories that break rotational symmetry, since the general solution for $q_{\alpha}^{i j}$ takes the form

$$
q_{\alpha}^{i j}=\beta_{\alpha}^{\nu} \tau_{\nu}^{i j}
$$

where $\alpha=1,2$. There are two invariant derivatives,

$$
D_{\alpha}=q_{\alpha}^{i j} \partial_{i} \partial_{j},
$$

and two corresponding gauge fields $a_{\alpha}$. The multipole algebra takes the form

$$
\left[T_{i}, \mathcal{P}_{1}^{I_{1}}\right]=\mathcal{P}_{0}, \quad\left[T_{i}, \mathcal{P}_{2}^{1}\right]=f_{i, I_{1}} \mathcal{P}_{1}^{I_{1}},
$$

where $f_{i, I_{1}}$ are the structure constants that are determined by $\mu_{i j}^{1}$.

Next we consider $k=2$. In this case there is a single invariant derivative and a single gauge field. After gauging the Gauss law eliminates all local gauge d.o.f. If the quadratic symmetries are both traceless, then the only allowed $q^{i j} \propto \delta^{i j}$ and the corresponding gauge field $a_{1}$ is the pure trace $a_{1}=\delta^{i j} a_{i j}$. This is reminiscent of the linearized dilaton coupling.

\section{Degenerate case}

A more interesting structure arises in the degenerate case. The multipole symmetry contains a single linear and single quadratic term, which take the form

$$
\mu^{I_{1}}=(m,-n), \quad \mu^{I_{2}}=\left(\begin{array}{cc}
m^{2} & -m n \\
-m n & n^{2}
\end{array}\right) .
$$

The corresponding polynomials are

$$
P_{1}^{1}=m x_{1}-n x_{2}, \quad P_{2}^{1}=\left(m x_{1}-n x_{2}\right)^{2} .
$$

The dipole moment $q^{i}$ has to be orthogonal to $\mu^{I_{1}}$ and be a null vector of $\mu^{I_{2}}$. To following vector satisfies these criteria:

$$
q_{1}^{i}=(n, m)
$$

Furthermore, there are two quadrupole matrices that satisfy Eq. (49), which are given explicitly by

$$
q_{2}^{i j}=\ell_{2}\left(\begin{array}{cc}
n & \frac{m}{2} \\
\frac{m}{2} & 0
\end{array}\right), \quad q_{3}^{i j}=\ell_{3}\left(\begin{array}{cc}
0 & \frac{n}{2} \\
\frac{n}{2} & m
\end{array}\right),
$$

where $\ell_{2}, \ell_{3}$ are the overall length scales.

The multipole algebra does not contain rotations, and takes a simple form,

$$
\begin{array}{cc}
{\left[\mathcal{P}_{1}^{1}, T_{\perp}\right]=\mathcal{P}_{0},} & {\left[\mathcal{P}_{2}^{I_{2}}, T_{\perp}\right]=\mathcal{P}_{1}^{1},} \\
{\left[\mathcal{P}_{1}^{1}, T_{\|}\right]=0,} & {\left[\mathcal{P}_{2}^{I_{2}}, T_{\|}\right]=0,}
\end{array}
$$

where $T_{\|}$and $T_{\perp}$ are translations in the direction parallel and perpendicular to $q^{i}$.

There are three invariant derivatives,

$$
D_{1}=q_{1}^{i} \partial_{i}, \quad D_{2}=q_{2}^{i j} \partial_{i} \partial_{j}, \quad D_{3}=q_{3}^{i j} \partial_{i} \partial_{j},
$$

and three corresponding gauge fields, $a_{\alpha}$. There is a nonlinear relation between the invariant derivatives, which takes the form

$$
D_{1}^{2}=n D_{2}+m D_{3}
$$

This relation allows us to include only the terms linear in $D_{1}$.

The Gauss law takes the form

$$
D_{\alpha}^{\dagger} e_{\alpha}=\rho .
$$

The corresponding charge configurations are illustrated in Fig. 2. Note that, due to the existence of the hopping 

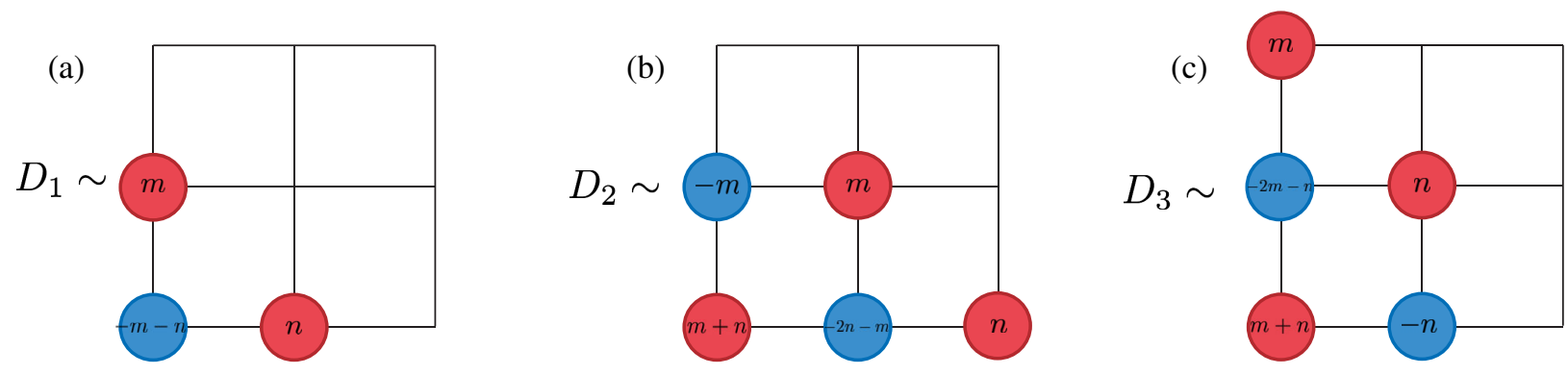

(d)

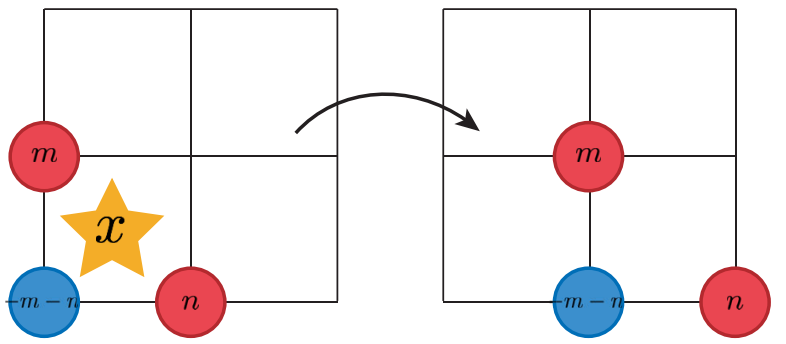

(e)

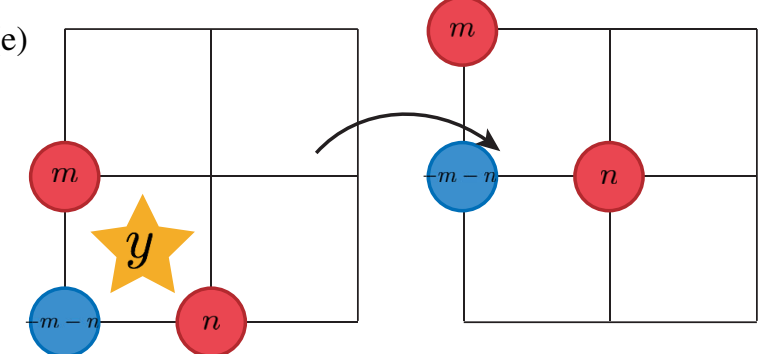

FIG. 2. (a)-(c) The elementary charge configurations, corresponding to $D_{\alpha}$, for the degenerate theory, characterized by Eqs. (62) and (63), with invariant derivatives given by Eq. (64). (d) Application of the charge configuration corresponding to $D_{2}$ results in hopping of the $(n, m)$ dipole in $x$ direction. (e) Application of the charge configuration corresponding to $D_{3}$ results in hopping of the $(n, m)$ dipole in $y$ direction.

operators for the $(n, m)$ dipole, the latter is fully mobile. However, the charges themselves are immobile.

\section{Magnetic fields}

To be complete, we construct a set of "magnetic fields." For the purpose of this work, we call a magnetic field any gauge-invariant combination of the gauge fields $a_{\alpha}$. Such construction turns out to be quite a nontrivial problem in general, because algebraic relations between the invariant derivatives lead to differential constraints between the magnetic fields (somewhat like the vanishing of divergence of a magnetic field in 3D electrodynamics). Although the 2D theories discussed here are somewhat trivial, as we clarify below, we keep the discussion general because a similar algebra of constraints arises in higher dimensions, notably for the U(1) Haah code discussed below.

To illustrate the subtlety, we first proceed in a naive way. By inspection, we find 4 magnetic fields,

$$
\begin{array}{r}
b_{1}=D_{2} a_{3}-D_{3} a_{2}, \quad b_{2}=D_{1} a_{3}-D_{3} a_{2}, \\
b_{3}=D_{1} a_{2}-D_{2} a_{1}, \quad b_{4}=D_{1} a_{1}-n a_{2}-m a_{3},
\end{array}
$$

where the first three are gauge invariant by virtue of the commutativity of the partial derivatives, while the latter is invariant due to the nonlinear relation Eq. (65). It turns out that these magnetic fields satisfy several constraints. These constraints can be obtained by applying various invariant derivatives to $b_{4}$. We find the following constraints:

$$
\begin{gathered}
D_{2} b_{4}+D_{1} b_{3}+m b_{1}=0, \\
D_{3} b_{4}+D_{1} b_{2}-n b_{1}=0, \\
D_{1} b_{4}+m b_{2}+n b_{3}=0 .
\end{gathered}
$$

These constraints are not independent. Indeed, taking a linear combination of the first two constraints, we find

$$
D_{1}\left(D_{1} b_{4}+m b_{2}+n b_{3}\right)=0,
$$

which, upon removing the derivative $D_{1}$, coincides with the last constraint Eq. (71). Thus, there are two independent components of magnetic field.

\section{Dynamics and Higgs terms}

Finally, we discuss the dynamics of the translationinvariant multipole 2D gauge theories. The degenerate theory discussed above is fully gapped. To see this we note that the combinations

$$
\beta_{1}=\partial_{x} a_{1}-a_{2}, \quad \beta_{2}=\partial_{y} a_{1}-a_{3}
$$

are gauge invariant. These magnetic fields are related to the magnetic fields introduced above as follows:

$$
\begin{gathered}
b_{4}=n \beta_{1}+m \beta_{2}, \quad b_{1}=-D_{1} \beta_{1}, \\
b_{2}=-D_{1} \beta_{2}, \quad b_{3}=-D_{2} \beta_{1}-D_{3} \beta_{1} .
\end{gathered}
$$


The low-energy Lagrangian takes the form

$$
\mathcal{L}=\sum_{\alpha} \dot{e}_{\alpha}^{2}+\beta_{1}^{2}+\beta_{2}^{2}
$$

We can choose the gauge, where $a_{1}=0$. In this gauge, $\beta_{1}^{2}$ and $\beta_{2}^{2}$ are mass terms for the $a_{2}$ and $a_{3}$ fields. Thus, at low energies, below the gap of $a_{2}$ and $a_{3}$, the degenerate 2D theory has no local d.o.f.

\section{F. Anisotropic scaling}

Next, we would like to investigate the scaling properties of the two-dimensional theories. We focus on the case when a single component of the dipole moment is conserved. The Lagrangian

$$
\mathcal{L}=\dot{\varphi} \dot{\varphi}+\sum_{\alpha} \lambda^{\alpha}\left(D_{\alpha} \varphi\right)^{2}
$$

does not possess any peculiar scaling properties for generic values of $m, n$ and the coupling constants. However, there are a few interesting cases to consider. To make the scaling properties apparent, we introduce the variables $q=q^{i} x_{i} /|q|$ and $r=\mu_{i}^{1} x^{i} /\left|\mu^{1}\right|$. In terms of these variables, the Lagrangian takes the form

$$
\mathcal{L}=\dot{\varphi} \dot{\varphi}+\lambda_{1}^{\prime}\left(\partial_{q} \varphi\right)^{2}+\lambda_{2}^{\prime}\left(\partial_{q}^{2} \varphi\right)^{2}+\lambda_{3}^{\prime}\left(\partial_{r} \partial_{q} \varphi\right)^{2},
$$

where $\lambda_{\alpha}^{\prime}$ are linear combinations of $\lambda_{\alpha}$, with the coefficients determined by $m, n$. The lack of the $\partial_{r}^{2} \varphi$ term is a manifestation of the degeneracy of the theory. If $\lambda_{2}^{\prime}=0$, then the theory exhibits the following scaling symmetry:

$$
t \rightarrow t, \quad q \rightarrow q, \quad r \rightarrow \beta r, \quad \varphi \rightarrow \beta^{-1 / 2} \varphi .
$$

This symmetry implies scale invariance in the direction of the conservation of the dipole moment. If such scaling symmetry is enforced, it leads to a relation between the coupling constants $\lambda_{2}=-\left(\mathrm{m}^{2} / \mathrm{n}^{2}\right) \lambda_{3}$.

Next, we relax the quadratic multipole symmetry. The multipole algebra, in this case, still closes and still does not contain the rotational symmetry since we have picked a preferred direction (namely, the conserved component of the dipole moment). For such a theory the Lagrangian is no longer degenerate and is given by Eq. (78) plus an extra term, $\lambda_{4}^{\prime}\left(\partial_{r}^{2} \varphi\right)^{2}$. Such a theory acquires an interesting scaling symmetry if we set $\lambda_{2}^{\prime}=\lambda_{3}^{\prime}=0$,

$$
\mathcal{L}_{\text {inv }}=\dot{\varphi} \dot{\varphi}+\lambda_{1}^{\prime}\left(\partial_{q} \varphi\right)^{2}+\lambda_{4}^{\prime}\left(\partial_{r}^{2} \varphi\right)^{2},
$$

where the scaling symmetry takes a highly anisotropic form:

$$
t \rightarrow \beta t, \quad q \rightarrow \beta q, \quad r \rightarrow \beta^{1 / 2} r, \quad \varphi \rightarrow \beta^{-1 / 4} \varphi .
$$

If such symmetry is enforced, only two charge configurations are allowed. We leave the detailed investigation of such symmetries to future work. Clearly, anisotropic scaling is possible only if the systems in question break rotational symmetry, which is often the case in condensed matter physics. Moreover, the anisotropic scaling implies the existence of a length scale in the long-distance effective theory, indicating a form of spatial order. Furthermore, theories with anisotropic scaling appear in the theories of liquid crystals [72,73] and nematic-smectic transitions [74]. It is possible that such symmetries naturally emerge close to a quantum critical point that exhibits dimensional reduction $[75,76]$.

\section{G. U(1) Haah code in three dimensions}

Next we turn to the U(1) Haah code studied in Ref. [66]. We start by postulating the symmetries

$$
\delta \varphi=\lambda+\lambda_{I_{1}}^{1} P_{1}^{I_{1}}+\lambda_{I_{2}}^{2} P_{2}^{I_{2}},
$$

where

$$
\begin{gathered}
P_{1}^{1}=x_{1}-x_{2}, \quad P_{1}^{2}=x_{1}+x_{2}-2 x_{3}, \\
P_{2}^{1}=\left(x_{1}-x_{2}\right)\left(x_{1}+x_{2}-2 x_{3}\right), \\
P_{2}^{2}=\left(2 x_{1}-x_{2}-x_{3}\right)\left(x_{2}-x_{3}\right) .
\end{gathered}
$$

The polynomials can also be represented by coefficient matrices as in Eqs. (16) and (17):

$$
\begin{array}{rlrl}
\mu_{i}^{1}=(1,-1,0), & \mu_{i}^{2}=(1,1-2), & \\
\mu_{i j}^{1}=\left(\begin{array}{ccc}
1 & 0 & -1 \\
0 & -1 & 1 \\
-1 & 1 & 0
\end{array}\right), & \mu_{i j}^{2}=\left(\begin{array}{ccc}
0 & 1 & -1 \\
1 & -1 & 0 \\
-1 & 0 & 1
\end{array}\right) .
\end{array}
$$

The multipole algebra takes exactly the form Eqs. (21)-(24). The dipole and quadrupole vectors are found by solving the constraints Eqs. (33)-(35). We find five solutions which are explicitly given by

$$
\begin{gathered}
\bar{q}_{1}^{i}=\bar{\ell}_{0}(1,1,1), \quad \bar{q}_{1}^{i j}=\bar{\ell}_{1}\left(\begin{array}{ccc}
1 & 0 & 0 \\
0 & 0 & -\frac{1}{2} \\
0 & -\frac{1}{2} & 0
\end{array}\right), \\
\bar{q}_{2}^{i j}=\bar{\ell}_{2}\left(\begin{array}{ccc}
0 & \frac{1}{2} & 0 \\
\frac{1}{2} & 0 & 0 \\
0 & 0 & -1
\end{array}\right), \quad \bar{q}_{3}^{i j}=\bar{\ell}_{3}\left(\begin{array}{ccc}
0 & 0 & \frac{1}{2} \\
0 & 0 & \frac{1}{2} \\
\frac{1}{2} & \frac{1}{2} & 1
\end{array}\right),
\end{gathered}
$$




$$
\bar{q}_{4}^{i j}=\bar{\ell}_{4}\left(\begin{array}{ccc}
0 & 0 & 0 \\
0 & 1 & \frac{1}{2} \\
0 & \frac{1}{2} & 1
\end{array}\right),
$$

where $\bar{\ell}_{\alpha}$ are the overall scales, among which $\bar{\ell}_{0}$ is dimensionless, so we set it to 1 . Recall that these scales cannot be determined from the constraints alone. To lighten up the equations we will set all $\bar{\ell}_{\alpha}=1$; however, the reader should be keenly aware that there is some freedom in overall scales. Thus we have five invariant derivatives:

$$
\bar{D}_{1}=\bar{q}^{i} \partial_{i}, \quad \bar{D}_{\alpha}=\bar{q}_{\alpha}^{i j} \partial_{i} \partial_{j}
$$

An invariant Lagrangian of the form Eq. (40) can be written already at this stage. It turns out, however, that such a Lagrangian is not invariant with respect to rotations in the $\mu_{i}^{1}-\mu_{j}^{2}$ plane. We can additionally enforce the invariance under rotations. Technically this is done by taking the linear combinations of the tensors $\bar{q}_{\alpha}^{i j}$ that are invariant under such rotations. There are two such linear combinations:

$$
\begin{aligned}
& q_{1}^{i j}=\bar{q}_{1}^{i j}+\bar{q}_{4}^{i j}=\left(\begin{array}{ccc}
1 & 0 & 0 \\
0 & 1 & 0 \\
0 & 0 & 1
\end{array}\right), \\
& q_{2}^{i j}=\bar{q}_{2}^{i j}+\bar{q}_{3}^{i j}=\left(\begin{array}{ccc}
0 & \frac{1}{2} & \frac{1}{2} \\
\frac{1}{2} & 0 & \frac{1}{2} \\
\frac{1}{2} & \frac{1}{2} & 0
\end{array}\right) .
\end{aligned}
$$

Thus we have three invariant derivatives that take the form

$$
D_{1}=\bar{D}_{1}, \quad D_{2}=q_{1}^{i j} \partial_{i} \partial_{j}, \quad D_{3}=q_{2}^{i j} \partial_{i} \partial_{j},
$$

while the covariant derivatives are given by $\nabla_{\beta} \varphi=$ $D_{\beta} \varphi+a_{\beta}$.

The most general Lagrangian, consistent with the (gauged) multipole algebra, takes the form

$\mathcal{L}=\nabla_{0} \varphi \nabla_{0} \varphi-g_{\alpha \beta}\left(\nabla_{\alpha} \varphi\right)\left(\nabla_{\beta} \varphi\right)+\cdots-\mathcal{H}[e, b]$

where the dots stand for the terms higher in derivatives, $g_{\alpha \beta}$ is the matrix of coupling constants, and $\mathcal{H}[e, b] \propto \sum_{\beta} e_{\beta}^{2}+$ $b_{1}^{2}+b_{2}^{2}$ is the Hamiltonian for the gauge fields. It is important to emphasize that some of the coupling constants have dimension of length. This reflects the presence of an emergent length scale, characteristic of layered and smectic phases [77].

The Gauss law takes the form

$$
\sum_{\beta} D_{\beta}^{\dagger} e_{\beta}=\rho .
$$

The elementary charge configurations are illustrated in Fig. 3.

Finally, we note that there is a nonlinear relation among the invariant derivatives, namely,

$$
\left(D_{1}\right)^{2}=D_{2}+2 D_{3}
$$

which corresponds to

$$
q^{i} \otimes q^{j}=q_{1}^{i j}+2 q_{2}^{i j}
$$

These relations reduce the number of independent higherorder terms and restrict the magnetic fields, but do not allow us to eliminate any of the invariant derivatives.

Next, we turn to the construction of the magnetic fields. As before, the latter are defined to be the gauge-invariant combinations of the gauge fields. There are 4 gaugeinvariant functions that we construct by inspection. These are given exactly by Eqs. (67) and (68) These magnetic fields are not all independent and satisfy the constraints Eqs. (69)-(71). We again find that only two components of the magnetic field are independent. In contrast to the 2D theory, there are no Higgs-type terms that we can write; thus, at low energy there are two gapless d.o.f. We further note that the presence of invariant derivatives of different degrees appears to be necessary for the presence of immobile particles.

The above theory includes the generalized gauge theory for the U(1) Haah code of Ref. [66]. It appears that in Ref. [66] the authors kept only the following invariant derivatives:

$$
D_{1}^{\mathrm{BB}}=D_{1}, \quad D_{2}^{\mathrm{BB}}=D_{3}-2 D_{1} .
$$

We are not aware of an additional symmetry principle that would force us to discard $D_{2}$. Since the Lagrangian Eq. (82) is effective, it must include all terms allowed by the general principles. The addition of an extra derivative and, therefore, an extra charge configuration does not contradict the conclusion about "fractal dynamics" observed in Eq. (65), as we discuss in the next section.

The Lagrangian Eq. (95) has a hidden sliding symmetry. To see it, we introduce new variables, $x=\mu_{i}^{1} x^{i} /\left|\mu^{1}\right|$ and $y=\mu_{i}^{2} x^{i} /\left|\mu^{2}\right|$. Then all invariant derivatives $D_{\alpha}$ (and consequently the Lagrangian) are also invariant under an infinite symmetry,

$$
\delta \varphi\left(z, \bar{z}, x_{3}\right)=f(z)+g(\bar{z}), \quad z=x+i y,
$$

where $f(z)$ is holomorphic and $g(\bar{z})$ is antiholomorphic. This is an example of a well-known "sliding" symmetry $[33,34,78]$ that appears in physics of smectics [79] and it can be understood as a continuous version of subsystems symmetries. This symmetry is responsible for an infinite number of conserved charges noticed in Ref. [66]. 
(a)

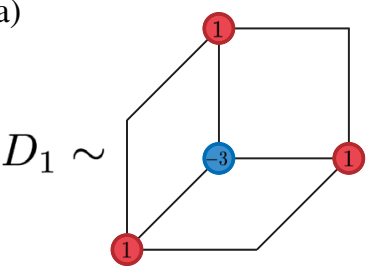

(b)

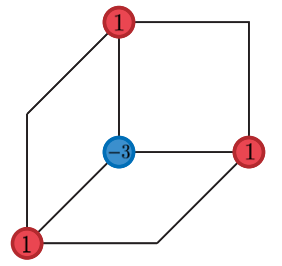

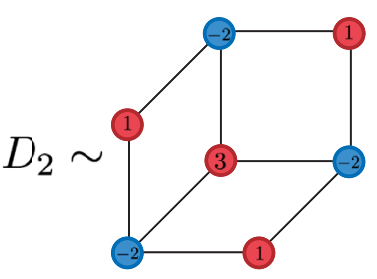

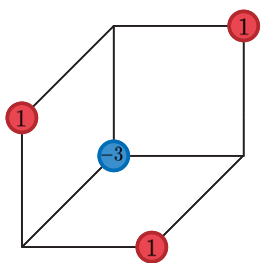

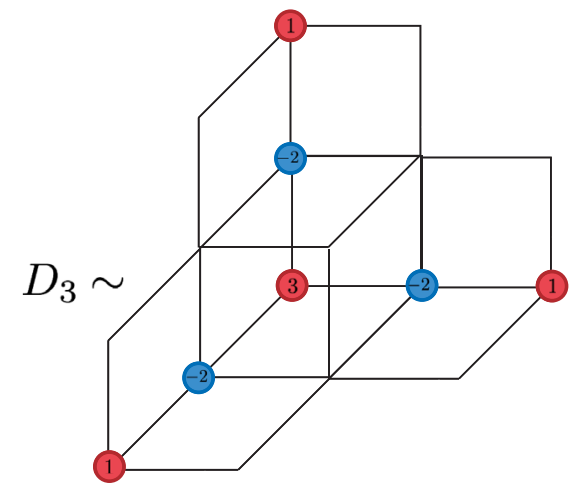

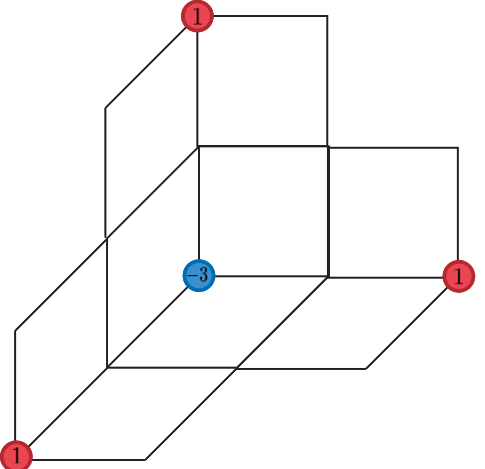

FIG. 3. (a) The elementary charge configurations, corresponding to $D_{\alpha}$, for the effective theory for the U(1) Haah code Eq. (94) charge configurations. (b) A different basis of elementary charge configurations. The first two configurations are precisely the ones studied in Ref. [66], while the last charge configuration is allowed by symmetries and is linearly independent from others.

Finally, the Lagrangian Eq. (95) exhibits an anisotropic scaling symmetry, which takes fthe orm

$$
\begin{aligned}
& t \rightarrow \lambda t, \quad x \rightarrow \lambda^{1 / 2} x, \quad y \rightarrow \lambda^{1 / 2} y, \quad x_{3} \rightarrow \lambda x_{3}, \\
& \varphi \rightarrow \lambda^{-1 / 2} \varphi .
\end{aligned}
$$

We leave the investigation of the physical consequences of this symmetry to future work.

\section{H. Coupling to charged matter}

In this section we consider charged matter, represented by a complex scalar field. This is not the most general situation, since the matter fields will not transform under rotations, but it will serve a good illustrative purpose. The inspiration for the following construction is taken from Refs. [20,65].

In the previous section we explained how to construct the invariant derivatives for an arbitrary charge-conserving multipole algebra. Those derivatives were used to couple a phase field $\varphi$ minimally to the multipole gauge fields $a_{\alpha}$. To introduce the charged matter we view the phase field as a phase of a charged scalar, according to

$$
\Phi=\sqrt{\rho} e^{i \varphi} .
$$

We will concentrate on the homogeneous multipole algebras. In this case the invariant derivatives are also homogeneous and can be ordered by the degree as follows:

$$
q_{\alpha}^{i} \partial_{i}, \quad q_{\alpha}^{i j} \partial_{i} \partial_{j}, \quad \ldots .
$$

The covariant derivatives of the complex scalar are defined according to

$$
\begin{gathered}
\mathcal{D}_{\alpha}^{1}[\Phi]=q_{\alpha}^{i} \partial_{i} \Phi-i a_{\alpha}, \\
\mathcal{D}_{\beta}^{2}[\Phi]=q_{\beta}^{i j}\left(\partial_{i} \Phi \partial_{j} \Phi-\Phi \partial_{i} \partial_{j} \Phi\right)-i a_{\beta}, \\
\ldots
\end{gathered}
$$

It immediately follows that using Eq. (102) in Eqs. (104) and (105) leads to the invariant derivatives acting on $\varphi$ that we discussed previously. The invariant Lagrangian then takes the form

$\mathcal{L}=\dot{\Phi}^{\dagger} \dot{\Phi}-g_{\alpha \beta}^{1} \mathcal{D}_{\alpha}^{1}[\Phi]^{\dagger} D_{\beta}^{1}[\Phi]-g_{\alpha \beta}^{2} \mathcal{D}_{\alpha}^{2}[\Phi]^{\dagger} D_{\beta}^{2}[\Phi]-\cdots$,

where the terms are arranged in such a way that global U(1) invariance is preserved and $g_{\alpha \beta}$ is a matrix of coupling constants. It is an open problem to construct an analogue of such formalism for inhomogeneous multipole algebras as well as Lagrangians invariant up to a total derivative.

\section{EXTENSIONS}

In this section we discuss two extensions of the present formalism. One extension includes the point group symmetries of the lattice, whereas the other one includes charge 
condensation. We also explain the relation between the present ideas and the formalism of polynomials over finite fields.

\section{A. Crystalline multipole algebra}

We have already observed that not all multipole algebras are consistent with continuous spatial rotations. We have also noted that the symmetry parameters have the dimension of length and, ultimately, have to be determined by the lattice constant. Keeping the lattice physics in mind, we can relax the rotational symmetry from continuous to a point group symmetry. Presently there is no general theory of the multipole algebras combined with the crystalline symmetries. Instead, we consider an example $-C_{4}$ symmetry in two spatial dimensions. The polynomial symmetries compatible with $C_{4}$ are

$\delta \varphi=\lambda \delta_{i j} x^{i} x^{j}+\lambda_{I_{4}} P_{4}^{I_{4}}(x), \quad P_{4}^{1}=x_{1}^{4}+x_{2}^{4}$,

$P_{4}^{2}=x_{1}^{3} x_{2}-x_{1} x_{2}^{3}, \quad P_{4}^{3}=x_{1}^{2} x_{2}^{2}$,

whereas if we were to require continuous rotational symmetry, we would find a single quartic polynomial $P_{4}=P_{4}^{1}+2 P_{4}^{3}=\left(x_{1}^{2}+x_{2}^{2}\right)^{2}$. Thus we find an interesting phenomenon: restricting continuous spatial symmetries to the crystalline ones (i.e., reducing the symmetry) allows for increasing the multipole symmetry. This will ultimately lead to more intricate constraints on the effective Lagrangian for the "spin-4" field.

\section{B. Charge condensation}

Lattice fracton models usually do not have a U(1) integer charge, rather they possess a $\mathbb{Z}_{p}$ symmetry, which means that the charge lattice is reduced to $\mathbb{Z} / p \mathbb{Z}$; i.e., the excitations of charge $p$ can disappear into vacuum and are equivalent to charge 0 . This constraint is particularly effective if we have already introduced a lattice.

In light of this possibility, we revisit the models from the previous section. We start with a two-dimensional model, characterized by the symmetry algebra Eq. (58). In this model condensation of charge- 2 and of charge- 3 objects leads to dramatically different macroscopic behavior. We consider the case when $n=m=1$. When charge- 2 objects are condensed, we can modify Fig. 2 to the $\mathbb{Z}_{2}$-valued charges, as illustrated in Fig. 4. The $(1,1)$ dipole configuration, corresponding to $D_{1}$, turns into an ordinary hopping operator, in the $(1,1)$ direction. The $\mathbb{Z}_{2}$ charges can be easily separated as shown in Fig. 5, but only along the $(1,1)$ direction. Thus, such charges are dimension-one particles, capable of hopping in the $(1,1)$ direction only.

In the $\mathbb{Z}_{3}$ case, when charge- $3 n$ objects are equivalent to vacuum, the $D_{1}$ charge configuration is no longer a hopping operator since $Q=-2 \sim Q=1$. The charge configurations now take form illustrated in Fig. 6.
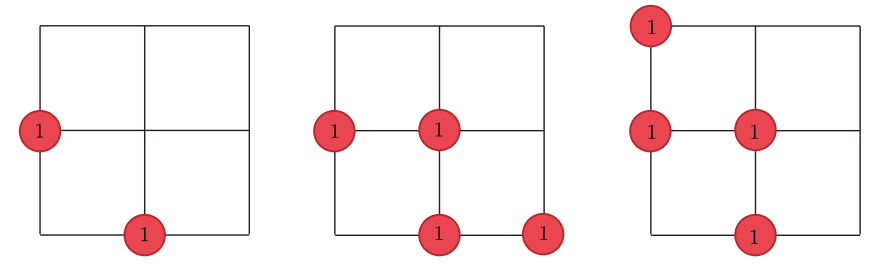

FIG. 4. Elementary charge configurations in the $\mathbb{Z}_{2}$ version of the theory described by Eq. (64). The first configuration is a hopping operator in the $(1,1)$ direction.

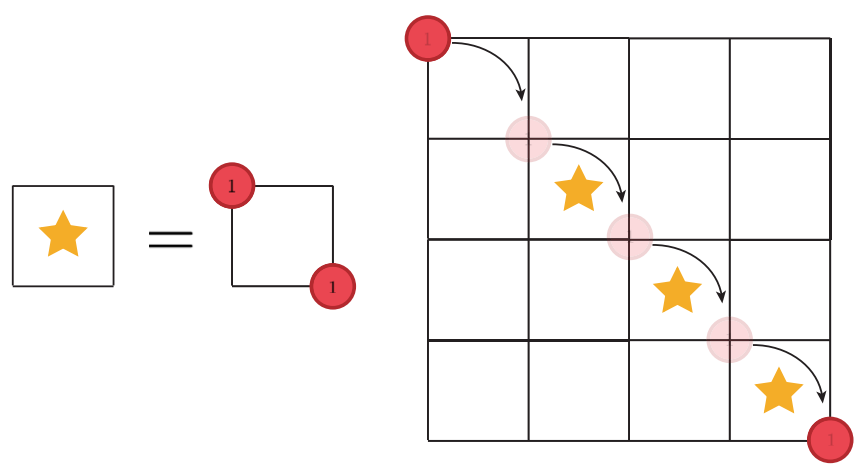

FIG. 5. Hopping operator in the $(1,-1)$ direction. This operator corresponds to the $D_{1}$ invariant derivative from the Eq. (64) after the charge- 2 condensation. Thus, a single charge is a dimensionone particle moving in the $(1,-1)$ direction.
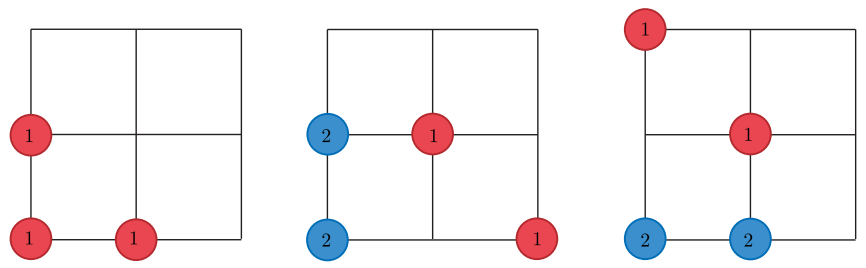

FIG. 6. Elementary charge configurations in the $\mathbb{Z}_{3}$ version of the degenerate $d=2$ theory Eq. (64). The operator, corresponding to $D_{1}$, is no longer a hopping operator. Note, however, that the $(1,1)$ dipole is fully mobile. This theory exhibits fractal operators.

If we try to separate the charges created by $e^{i a_{1}}$, we find a fractal structure (see Fig. 7), which corresponds to the $\mathbb{Z}_{3}$ version of the Sierpinski triangle. We note, however that according to the general results of Refs. [3,80], such theories cannot be topologically ordered. Meaning that they are not stable to perturbations that break the multipole symmetry. Rather, they should be viewed as Symmetry-protected topological phases (SPTs).

Next, we turn to the U(1) Haah code in three dimensions. In the previous section we found that any charge configuration is generated by a combination of 3 basis charge configurations. At first sight this remains true after the condensation of charge-2 excitations (see Fig. 8). In the original version of Haah's code, which is based on the $\mathbb{Z}_{2}$ charge, there are only 2 charge configurations. 


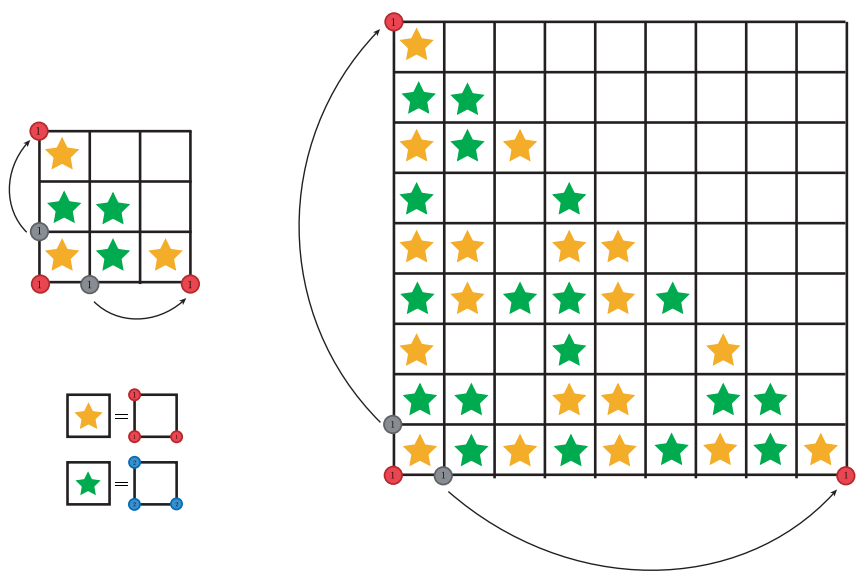

FIG. 7. The $(1,1)$ dipole is created in the left-hand bottom corner. The charges can be hopped by applying an operator living on a fractal structure. Yellow stars correspond to the operator $D_{1}$, while green stars correspond to the operator $2 D_{1}$. It is clear that the ability to separate charges becomes sensitive to the system size since the linear size of the operators is $3^{k}$. The next hopping operator will be of the size 27 . Both charge- 1 and charge- 2 excitations can be hopped using similar operators. To hop charge-2 excitations one must replace all green stars by the yellow ones and vice versa.

The two facts are reconciled by observing that after charge- 2 condensation one of the charge configurations can be eliminated via applying the configuration corresponding to $D_{1}$ multiple times, as shown in Fig. 8. In the $\mathbb{Z}_{3}$ version, we find three independent charge configurations.

(a)

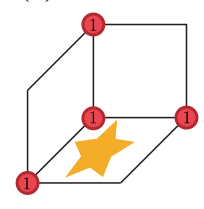

(b)

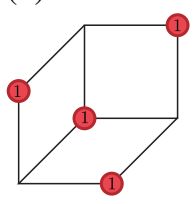

(c)

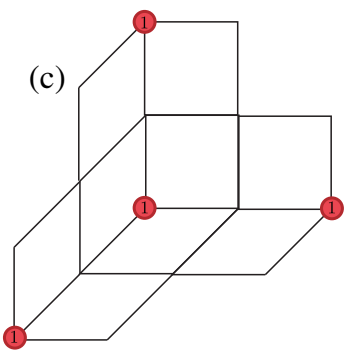

d)

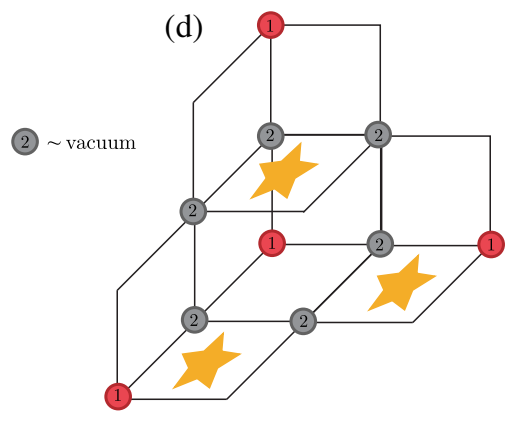

FIG. 8. (a)-(c). Charge configurations in the $\mathbb{Z}_{2}$ Haah code. (d) The (c) configuration can be obtained as a combination of (a) configurations applied to plaquettes labeled by the star.

\section{Polynomials over finite fields}

Some of the original works on fractal phases $[3,5,80]$ use the language of polynomials over the finite fields. In the present case, the "creation operators" originate in the structure of the invariant derivatives. The latter become differential operators with the coefficients in the same finite field upon the charge condensation. In this section we describe the relation between the formalism of polynomials over finite fields and the field-theoretic approach.

To get some intuition about the possible relation, we convert the graphical representation of charge configurations into polynomials as explained in Refs. $[3,5,80]$. In this construction one considers formal multivariate polynomials over a finite field, say, $\mathbb{Z}_{p}$, for a prime $p$ [or over $\mathbb{Z}$ in the $\mathrm{U}(1)$ case]. The coefficients of the polynomials give the values of charges, while the powers of formal variables provide the coordinates. For example, $q x^{n} y^{m}$ corresponds to a charge $q$ located at position $(n, m)$.

Consider the two-dimensional dipole $q^{i}=(1,1)$ case discussed previously. The basis charge configurations of Fig. 6 correspond to the following polynomials (the coefficients are in $\mathbb{Z}_{3}$ ):

$$
\begin{gathered}
\mathcal{H}_{1}=x+y+1, \quad \mathcal{H}_{2}=x^{2}+x y+2 y+2, \\
\mathcal{H}_{3}=y^{2}+x y+2 x+2 .
\end{gathered}
$$

Polynomials $\mathcal{H}_{2}$ and $\mathcal{H}_{3}$ are divisible by $\mathcal{H}_{1}$ over $\mathbb{Z}_{3}$. The coefficients in the polynomials sum up to $0 \bmod 3$, which reflects the conservation of charge mod3. The polynomials $\mathcal{H}_{i}$ satisfy the same relation as the invariant derivatives Eq. (65):

$$
\mathcal{H}_{1}^{2}=\mathcal{H}_{2}+\mathcal{H}_{3}
$$

The use of these polynomials guarantees that all charge configurations will satisfy the conservation laws.

The hopping of the dipole can be implemented in two different ways: additively and multiplicatively. The latter is accomplished via multiplication of the polynomial by either $x$ or $y$. Indeed, $x \mathcal{H}_{1}$ and $y \mathcal{H}_{1}$ correspond to the $(1,1)$ dipoles that hopped either in the $x$ or in the $y$ direction. The "additive" hopping polynomials can be constructed as $-\mathcal{H}_{1}+x \mathcal{H}_{1}=2 \mathcal{H}_{1}+x \mathcal{H}_{1}=\mathcal{H}_{2}$. Then, indeed, $\mathcal{H}_{1}+$ $\mathcal{H}_{2}=x \mathcal{H}_{1}$. Note that it is the additive hopping operators that correspond to the charge configurations; see Fig. 6. Since all the polynomials have a common factor, $x+y+1$, it follows that the configuration corresponding to this common factor is mobile.

Monomials $x$ or $y$ themselves are not allowed due to charge conservation. The pair creation, corresponding to $x+2 y$ or $y+2 x$, is also not allowed by the conservation of the dipole moment. The first allowed process is the triple creation, corresponding to $x+y+1$. This triple combination is mobile since the operators which hop it are also 
allowed by the conservation laws. The fractal operators of Fig. 7 are constructed by taking the powers $\mathcal{H}_{1}^{3 k}$. It appears that there is enough information in the field theory to construct all of the polynomial and fractal structure after specifying the lattice and the condensation process. Alternatively, it should be possible to arrive to the same set of polynomials imposing the constraints (i.e., multipole moment "conservation") directly in the polynomial language.

The Haah code is specified by similar data. In the $\mathbb{Z}_{2}$ case the relevant polynomials are

$$
\begin{gathered}
\mathcal{H}_{1}=x+y+z+1, \quad \mathcal{H}_{2}=x^{2}+y^{2}+z^{2}+1, \\
\mathcal{H}_{3}=x y+y z+x z+1 .
\end{gathered}
$$

The redundancy of one of the charge configurations, in the $\mathbb{Z}_{2}$ case discussed in the previous section, corresponds to a relation $\mathcal{H}_{1}^{2}=\mathcal{H}_{2}$. One way to understand this nonlinear relation is to note that in Eq. (97) multiplication by 2 is the same as multiplication by 0 over $\mathbb{Z}_{2}$ (which is no longer true over $\mathbb{Z}_{3}$ ). This time, however, there are no mobile operators since multiplication by $x, y$, or $z$ takes us outside of the allowed set of polynomials. The fractal structures are generated by $\mathcal{H}_{1}$ to various powers. We also note that the polynomials can be read out almost exactly from the invariant derivatives $D_{\alpha}$ and satisfy the same algebraic constraints. It would be interesting to see how much of the machinery of Ref. [3] can be phrased in terms of the derivative operators.

\section{Multipole moments over finite fields}

General multipole moments can be constructed using the formal derivatives over finite fields. We will illustrate the construction on the example of quadratic polynomials. Consider a polynomial,

$$
\mathcal{H}(x)=\sum_{i \leq j} h^{i j} x_{i} x_{j}+h^{i} x_{i}+h_{0},
$$

with the coefficient either in $\mathbb{Z}_{p}$ for a prime $p$ or in $\mathbb{Z}$. The total charge of the corresponding charge configuration is given by the sum of the coefficients within the appropriate field:

$$
Q[\mathcal{H}]=\mathcal{H}\left(\left\{x_{i}\right\}=1\right)=\sum_{i \leq j} h^{i j}+\sum_{i} h^{i}+h^{0} .
$$

The conservation of charge states that we consider the polynomials satisfy $\mathcal{H}\left(\left\{x_{i}\right\}=1\right)=0$.

The dipole moment can be evaluated as follows. Note that the power of a monomial indicates position of the charge. Thus, to get the value of the position we have to take a derivative. To this end, we construct a vector of polynomials:

$$
\mathcal{D}^{k}=\partial^{k} \mathcal{H}=2 h^{k k} x_{k}+\sum_{j \neq k} h^{k j} x_{j}+h^{k} .
$$

The dipole moment is determined by summing the coefficients in every component within the field. The sum over coefficients is formally evaluated by setting $\left\{x_{i}\right\}=1$ :

$$
d^{k}[\mathcal{H}]=\mathcal{D}^{k}\left(\left\{x_{i}\right\}=1\right)=2 h^{k k}+\sum_{j} h^{k j}+h^{k} .
$$

One has to be careful with the "multiplication." The symbol $2 h^{k k}$ really means $2 h^{k k} \equiv h^{k k}+h^{k k} \bmod p$. This definition automatically allows us to mod out by the equivalence relations between the values of the dipole moment. We now give an example of such relations in the case of $\mathbb{Z}_{2}$. Consider a charge configuration $\mathcal{H}=x^{2}+x y+y^{2}+1$. The total charge is 0 over $\mathbb{Z}_{2}$ (and would be 4 over $\mathbb{Z}$ ). The dipole polynomial takes the form

$\overrightarrow{\mathcal{D}}=\left(\begin{array}{l}2 x+y \\ 2 y+x\end{array}\right)=\left(\begin{array}{l}y \\ x\end{array}\right) \Rightarrow \vec{d}[\mathcal{H}]=\left(\begin{array}{l}1 \\ 1\end{array}\right)=\left(\begin{array}{l}3 \\ 3\end{array}\right)$.

The latter equivalence between the dipole moments is a consequence of the fact that charge- 2 excitations can be pulled out of the vacuum and shift the total dipole moment by $\delta \vec{d}=\left(\begin{array}{c}2 n \\ 2 m\end{array}\right)$.

The arbitrary $k$ th multipole moment of the charge density can be defined in a similar fashion, if we restrict ourselves to configuration with vanishing lower moments. For example, the quadrupole moment is constructed from the matrix of second derivatives. In general, we have for the $k$ th moment $Q^{i_{1} i_{2} \ldots i_{k}}$ :

$$
\begin{gathered}
\mathcal{Q}^{i_{1} i_{2} \ldots i_{k}}=\partial^{i_{1}} \partial^{i_{2}} \ldots \partial^{i_{k}} \mathcal{H}, \\
\mathcal{Q}^{i_{1} i_{2} \ldots i_{k}}=\mathcal{Q}^{i_{1} i_{2} \ldots i_{k}}\left(\left\{x_{i}\right\}=1\right) .
\end{gathered}
$$

To check that these relations are true, one has to (i) construct the $k$ th moment according to the usual definition and (ii) use the constraints that all lower moments vanish. In the case when some of the lower moments are nonzero, the construction has to be appended.

Finally, we would like to demonstrate that the multipole moments so defined are consistent with the multiplicative realization of the translations. For brevity we demonstrate this on the example of the second moment. Translation in the $l$ th direction by $n$ lattice spacings is realized via multiplication by $x_{l}^{n}$. The change in the second moment is then given by

$$
\begin{aligned}
\delta_{l} \mathcal{Q}^{i j}= & \partial^{i} \partial^{j}\left(x_{l}^{n} \mathcal{H}\right)-\partial^{i} \partial^{j} \mathcal{H} \\
= & \delta_{l}^{i} \partial^{j} \mathcal{H}+\delta_{l}^{j} \partial^{i} \mathcal{H}+n(n-1) \delta_{l}^{i} \delta_{l}^{j} \mathcal{H} \\
& +\left(x_{l}^{n}-1\right) \partial^{i} \partial^{j} \mathcal{H} ;
\end{aligned}
$$


evaluating $\delta_{l} \mathcal{Q}^{i j}$ at $\left\{x_{i}\right\}=1$, we find

$$
\delta_{l} Q^{i j}=n \delta_{l}^{i} d^{j}+n \delta_{l}^{j} d^{i}+n(n-1) \delta_{l}^{i} \delta_{l}^{j} Q=0 .
$$

This variation vanishes provided all lower moments- total dipole and total charge in the present case-vanish. In the language of polynomials over the finite fields, the conservation laws are implemented as brute force constraints on various moments of the charge density. It is not clear how to introduce the finite field version of the polynomial shift symmetries.

\section{CONCLUSIONS AND DISCUSSIONS}

\section{A. Conclusions}

We have introduced the multipole algebra-an extension of space(time) symmetries that enforce conservation of certain multipole moments of the charge density. This algebra contains both spatial symmetries and, in the simplest scalar representation, the polynomial shift symmetries. We have explained how to gauge the latter in the flat space and have shown that the corresponding gauge theory satisfies a set of Gauss law constraints. These constraints imply that the local excitations correspond to certain charge configurations with prescribed moments of the charge density. In such models one encounters a difficulty in trying to separate the $\mathrm{U}(1)$ charges away form each other. Recently studied symmetric tensor gauge theories of various kinds naturally emerge from this structure and correspond to the maximally symmetric homogeneous multipole algebras. Crucially, the (gapless versions of) type-II fracton models also fall into the same category, and correspond to the less symmetric homogeneous multipole algebras.

We have discussed several concrete examples of the multipole algebras. The U(1) version of the Haah code fits naturally into this structure. Upon charge condensation from $\mathrm{U}(1)$ to $\mathbb{Z}_{2}$, we find exactly the charge configurations considered in the original work by Haah. It was found that for $\mathbb{Z}_{3}$ charges there is an additional basis charge configuration that cannot be ruled out on the basis of the symmetry alone. We have also discussed a two-dimensional example where the fractal structures naturally emerge upon the charge condensation from $\mathrm{U}(1)$ to $\mathbb{Z}_{3}$. Such $2 \mathrm{D}$ theories are gapped, but not topologically ordered. Thus, such theories should be viewed as SPTs of the multipole symmetry. Finally, an explanation relating the present construction to the formalism of polynomials over finite fields was provided.

\section{B. Discussions}

In this final part, we discuss some open problems remaining after this work. First and foremost, we were able to formulate a general structure which, upon charge condensation may (or may not) lead to the fractal operators. It is important to find the necessary and sufficient conditions, in the field theory language, for the appearance of these operators. Currently, we can establish their presence only by inspection.

As our two-dimensional example illustrates, it is possible to have fractal operators without topological order. Such theories are not stable to perturbations that break the multipole algebra. At the same time, we were able to reproduce the $\mathbb{Z}_{2}$ Haah code within the same framework. The latter, however, is topologically ordered and is stable against local perturbations, including the ones that break the multipole symmetry. It is not clear how to establish the existence of the topological order without going into details and comparing with the known commuting projector models.

The polynomial symmetries discussed above are clearly well defined on an infinite plane. When the system is placed on a torus, i.e., is subject to the periodic boundary conditions, the polynomial symmetries become inconsistent with the boundary conditions. If the field $\varphi$ is assumed to be compact, then we need to ensure that the exponents of these polynomials, $e^{i P_{a}^{I_{a}}}$, are consistent with the boundary conditions. Restricting to such polynomials will lead to the reduction in the number of symmetries. It would be interesting to see if identifying such polynomials provides the information about degeneracy on a torus as well as an indicator that signals whether the "Higgsed" theory is topologically ordered or not.

On a more formal field theory side, it would be interesting to develop a general procedure that allows gauging of the entire multipole algebra. Such gauging should lead to very exotic theories of gravity and/or elasticity. Partial progress on this topic has been made in regards to gauging the Bargmann algebra, which we have encountered upon studying traceless scalar charge theory [68]. It will also be interesting to understand how the multipole algebra manifests itself in the theory of elasticity and its dual gauge theory along the ideas of Ref. [81]. We plan to address these and other questions in a forthcoming work.

\section{ACKNOWLEDGMENTS}

It is a pleasure to thank A. Abanov, P. Hořava, and $\mathrm{J}$. Moore for stimulating discussions, and especially J. Haah for patiently explaining his work. I also would like to thanks M. Barkeshli, D. Bulmash, F. Burnell, S. Sondhi, and Y. You for the comments on the early version of the manuscript. A. G. was supported by Quantum Materials program at LBNL, funded by the U.S. Department of Energy under Contract No. DE-AC02-05CH11231 and by the Brown University start-up funds.

[1] That cannot be created by a local operator.

[2] C. Chamon, Quantum Glassiness in Strongly Correlated Clean Systems: An Example of Topological Overprotection, Phys. Rev. Lett. 94, 040402 (2005). 
[3] J. Haah, Local Stabilizer Codes in Three Dimensions without String Logical Operators, Phys. Rev. A 83, 042330 (2011).

[4] S. Bravyi, B. Leemhuis, and B. M. Terhal, Topological Order in an Exactly Solvable 3D Spin Model, Ann. Phys. (Amsterdam) 326, 839 (2011).

[5] B. Yoshida, Exotic Topological Order in Fractal Spin Liquids, Phys. Rev. B 88, 125122 (2013).

[6] S. Vijay, J. Haah, and L. Fu, A New Kind of Topological Quantum Order: A Dimensional Hierarchy of Quasiparticles Built from Stationary Excitations, Phys. Rev. B 92, 235136 (2015).

[7] S. Vijay, J. Haah, and L. Fu, Fracton Topological Order Generalized Lattice Gauge Theory, and Duality, Phys. Rev. B 94, 235157 (2016).

[8] System size-dependent degeneracy has appeared in the quantum Hall context previously [9].

[9] A. Kol and N. Read, Fractional Quantum Hall Effect in a Periodic Potential, Phys. Rev. B 48, 8890 (1993).

[10] S. Alexander and R. Orbach, Density of States on Fractals: Fractons, J. Phys. (Paris), Lett. 43, 625 (1982).

[11] M. Pretko, Generalized Electromagnetism of Subdimensional Particles: A Spin Liquid Story, Phys. Rev. B 96, 035119 (2017).

[12] M. Pretko, Subdimensional Particle Structure of Higher Rank U(1) Spin Liquids, Phys. Rev. B 95, 115139 (2017).

[13] M. Pretko, Emergent Gravity of Fractons: Mach's Principle Revisited, Phys. Rev. D 96, 024051 (2017).

[14] C. Xu, Novel Algebraic Boson Liquid Phase with Soft Graviton Excitations, arXiv:cond-mat/0602443.

[15] C. Xu and P. Hořava, Emergent Gravity at a Lifshitz. Point from a Bose Liquid on the Lattice, Phys. Rev. D 81, 104033 (2010).

[16] D. Bulmash and M. Barkeshli, Higgs Mechanism in HigherRank Symmetric U(1) Gauge Theories, Phys. Rev. B 97, 235112 (2018).

[17] H. Ma, M. Hermele, and X. Chen, Fracton Topological Order from Higgs and Partial Confinement Mechanisms of Rank-Two Gauge Theory, Phys. Rev. B 98, 035111 (2018).

[18] M. Pretko and L. Radzihovsky, Fracton-Elasticity Duality, Phys. Rev. Lett. 120, 195301 (2018).

[19] A. Gromov, Fractional Topological Elasticity and Fracton Order, Phys. Rev. Lett. 122, 076403 (2019).

[20] A. Kumar and A. C. Potter, Symmetry Enforced Fractonicity and Two-Dimensional Quantum Crystal Melting, Phys. Rev. B 100, 045119 (2019).

[21] M. Pretko and L. Radzihovsky, Symmetry Enriched Fracton Phases from Supersolid Duality, Phys. Rev. Lett. 121, 235301 (2018).

[22] H. Kleinert, Duality Transformation for Defect Melting, Phys. Lett. A 91, 295 (1982).

[23] H. Kleinert, Dual Model for Dislocation and Disclination Melting, Phys. Lett. A 96, 302 (1983).

[24] K. Slagle, A. Prem, and M. Pretko, Symmetric Tensor Gauge Theories on Curved Spaces, arXiv:1807.00827.

[25] K. Slagle and Y. B. Kim, Quantum Field Theory of X-Cube Fracton Topological Order and Robust Degeneracy from Geometry, Phys. Rev. B 96, 195139 (2017).
[26] K. Slagle and Y.B. Kim, X-Cube Model on Generic Lattices: New Phases and Geometric Order, Phys. Rev. B 97, 165106 (2018).

[27] W. Shirley, K. Slagle, Z. Wang, and X. Chen, Fracton Models on General Three-Dimensional Manifolds, Phys. Rev. X 8, 031051 (2018).

[28] W. Shirley, K. Slagle, and X. Chen, Foliated Fracton Order from Gauging Subsystem Symmetries, SciPost Phys. 6, 041 (2019).

[29] D. J. Williamson, Fractal Symmetries: Ungauging the Cubic Code, Phys. Rev. B 94, 155128 (2016).

[30] T. Devakul, Y. You, F. J. Burnell, and S. L. Sondhi, Fractal Symmetric Phases of Matter, SciPost Phys. 6, 007 (2019).

[31] Y. You, T. Devakul, F. J. Burnell, and S. L. Sondhi, Subsystem Symmetry Protected Topological Order, Phys. Rev. B 98, 035112 (2018).

[32] D. J. Williamson, Z. Bi, and M. Cheng, Fractonic Matter in Symmetry-Enriched U(1) Gauge Theory, arXiv:1809.10275.

[33] M. J. Lawler and E. Fradkin, Quantum Hall Smectics, Sliding Symmetry, and the Renormalization Group, Phys. Rev. B 70, 165310 (2004).

[34] Z. Nussinov and E. Fradkin, Discrete Sliding Symmetries, Dualities, and Self-Dualities of Quantum Orbital Compass Models and $p+i p$ Superconducting Arrays, Phys. Rev. B 71, 195120 (2005).

[35] Y. You, T. Devakul, F. J. Burnell, and S. L. Sondhi, Symmetric Fracton Matter: Twisted and Enriched, arXiv: 1805.09800 .

[36] A. Prem, M. Pretko, and R. Nandkishore, Emergent Phases of Fractonic Matter, Phys. Rev. B 97, 085116 (2018).

[37] A. Prem, S.-J. Huang, H. Song, and M. Hermele, Cage-Net Fracton Models, Phys. Rev. X 9, 021010 (2019).

[38] H. Ma, E. Lake, X. Chen, and M. Hermele, Fracton Topological Order via Coupled Layers, Phys. Rev. B 95, 245126 (2017).

[39] H. Ma, A. T. Schmitz, S. A. Parameswaran, M. Hermele, and R. M. Nandkishore, Topological Entanglement Entropy of Fracton Stabilizer Codes, Phys. Rev. B 97, 125101 (2018).

[40] O. Petrova and N. Regnault, A Simple Anisotropic ThreeDimensional Quantum Spin Liquid with Fracton Topological Order, Phys. Rev. B 96, 224429 (2017).

[41] H. Yan, Fracton Topological Order and Holography, Phys. Rev. B 99, 155126 (2019).

[42] S. Pai, M. Pretko, and R. M. Nandkishore, Localization in Fractonic Random Circuits, Phys. Rev. X 9, 021003 (2019).

[43] A. Prem, S. Vijay, Y.-Z. Chou, M. Pretko, and R. M. Nandkishore, Pinch Point Singularities of Tensor Spin Liquids, Phys. Rev. B 98, 165140 (2018).

[44] T. Devakul, S. A. Parameswaran, and S. L. Sondhi, Correlation Function Diagnostics for Type-I Fracton Phases, Phys. Rev. B 97, 041110(R) (2018).

[45] T. Devakul, Classifying Local Fractal Subsystem Symmetry Protected Topological Phases, Phys. Rev. B 99, 235131 (2019).

[46] M. Pretko, Higher-Spin Witten Effect and Two-Dimensional Fracton Phases, Phys. Rev. B 96, 125151 (2017).

[47] D. Bulmash and T. Iadecola, Braiding and Gapped Boundaries in Fracton Topological Phases, Phys. Rev. B 99, 125132 (2019). 
[48] K. Slagle, D. Aasen, and D. Williamson, Foliated Field Theory and String-Membrane-Net Condensation Picture of Fracton Order, SciPost Phys. 6, 043 (2019).

[49] R. M. Nandkishore and M. Hermele, Fractons, Annu. Rev. Condens. Matter Phys. 10, 295 (2019).

[50] D. T. Son, Newton-Cartan Geometry and the Quantum Hall Effect, arXiv:1306.0638.

[51] M. Geracie, D. T. Son, C. Wu, and S.-F. Wu, Spacetime Symmetries of the Quantum Hall Effect, Phys. Rev. D 91, 045030 (2015).

[52] A. Gromov and A. G. Abanov, Thermal Hall Effect and Geometry with Torsion, Phys. Rev. Lett. 114, 016802 (2015).

[53] B. Bradlyn and N. Read, Low-Energy Effective Theory in the Bulk for Transport in a Topological Phase, Phys. Rev. B 91, 125303 (2015).

[54] F. D. M. Haldane, Geometrical Description of the Fractional Quantum Hall Effect, Phys. Rev. Lett. 107, 116801 (2011).

[55] Y. You, G. Y. Cho, and E. Fradkin, Theory of Nematic Fractional Quantum Hall States, Phys. Rev. X 4, 041050 (2014).

[56] J. Maciejko, B. Hsu, S. A. Kivelson, Y. J. Park, and S. L. Sondhi, Field Theory of the Quantum Hall Nematic Transition, Phys. Rev. B 88, 125137 (2013).

[57] S. Golkar, D. X. Nguyen, and D. T. Son, Spectral Sum Rules and Magneto-Roton as Emergent Graviton in Fractional Quantum Hall Effect, J. High Energy Phys. 01 (2016) 021.

[58] S. Golkar, D. X. Nguyen, M. M. Roberts, and D. T. Son, Higher-Spin Theory of the Magnetorotons, Phys. Rev. Lett. 117, 216403 (2016).

[59] A. Gromov and D. T. Son, Bimetric Theory of Fractional Quantum Hall States, Phys. Rev. X 7, 041032 (2017).

[60] D. X. Nguyen, A. Gromov, and D. T. Son, Fractional Quantum Hall Systems Near Nematicity: Bimetric Theory, Composite Fermions, and Dirac Brackets, Phys. Rev. B 97, 195103 (2018).

[61] A. Nicolis, R. Rattazzi, and E. Trincherini, Galileon as a Local Modification of Gravity, Phys. Rev. D 79, 064036 (2009).

[62] T. Griffin, K. T. Grosvenor, P. Hořava, and Z. Yan, Multicritical Symmetry Breaking and Naturalness of Slow NambuGoldstone Bosons, Phys. Rev. D 88, 101701(R) (2013).

[63] K. Hinterbichler and A. Joyce, Goldstones with Extended Shift Symmetries, Int. J. Mod. Phys. D 23, 1443001 (2014).

[64] T. Griffin, K. T. Grosvenor, P. Hořava, and Z. Yan, Scalar Field Theories with Polynomial Shift Symmetries, Commun. Math. Phys. 340, 985 (2015).
[65] M. Pretko, The Fracton Gauge Principle, Phys. Rev. B 98, 115134 (2018).

[66] D. Bulmash and M. Barkeshli, Generalized U(1) Gauge Field Theories and Fractal Dynamics, arXiv:1806.01855.

[67] E. Lake, Higher-Form Symmetries and Spontaneous Symmetry Breaking, arXiv:1802.07747.

[68] R. Andringa, E. Bergshoeff, S. Panda, and M. De Roo, Newtonian Gravity and the Bargmann Algebra, Classical Quantum Gravity 28, 105011 (2011).

[69] E. S. Fradkin and M. A. Vasiliev, Candidate for the Role of Higher-Spin Symmetry, Ann. Phys. (N.Y.) 177, 63 (1987).

[70] This choice excludes the vector charge theories. The latter are obtained by choosing $\varphi$ to be a vector under the rotations.

[71] This is so because we are considering the shift symmetries, which are polynomial in the spatial coordinates, and not in time.

[72] J. Prost, The Physics of Liquid Crystals (Oxford University Press Inc., New York, 1995), Vol. 83.

[73] A. J. Beekman, J. Nissinen, K. Wu, K. Liu, R.-J. Slager, Z. Nussinov, V. Cvetkovic, and J. Zaanen, Dual Gauge Field Theory of Quantum Liquid Crystals in Two Dimensions, Phys. Rep. 683, 1 (2017).

[74] K. Sun, B. M. Fregoso, M. J. Lawler, and E. Fradkin, Fluctuating Stripes in Strongly Correlated Electron Systems and the Nematic-Smectic Quantum Phase Transition, Phys. Rev. B 78, 085124 (2008).

[75] C. Xu and J. E. Moore, Reduction of Effective Dimensionality in Lattice Models of Superconducting Arrays and Frustrated Magnets, Nucl. Phys. B716, 487 (2005).

[76] S. E. Sebastian, N. Harrison, C. D. Batista, L. Balicas, M. Jaime, P. A. Sharma, N. Kawashima, and I. R. Fisher, Dimensional Reduction at a Quantum Critical Point, Nature (London) 441, 617 (2006).

[77] P. M. Chaikin, T. C. Lubensky, and T. A. Witten, Principles of Condensed Matter Physics (Cambridge University Press, Cambridge, England, 1995), Vol. 1.

[78] D. G. Barci, E. Fradkin, S. A. Kivelson, and V. Oganesyan, Theory of the Quantum Hall Smectic Phase. I. Low-Energy Properties of the Quantum Hall Smectic Fixed Point, Phys. Rev. B 65, 245319 (2002).

[79] C. S. O'Hern and T. C. Lubensky, Nonlinear Elasticity of the Sliding Columnar Phase, Phys. Rev. E 58, 5948 (1998).

[80] J. Haah, Commuting Pauli Hamiltonians as Maps between Free Modules, Commun. Math. Phys. 324, 351 (2013).

[81] H. Kleinert, Double Gauge Theory of Stresses and Defects, Phys. Lett. A 97, 51 (1983). 